\title{
TOPOLOGICAL ENTROPY OF TRANSITIVE DENDRITE MAPS
}

\author{
VLADIMÍR ŠPITALSKÝ
}

\begin{abstract}
We show that every dendrite $X$ satisfying the condition that no subtree of $X$ contains all free arcs admits a transitive, even exactly Devaney chaotic map with arbitrarily small entropy. This gives a partial answer to a question of Baldwin from 2001.
\end{abstract}

\section{INTRODUCTION}

Let $X$ be a compact metric space. By a dynamical system on $X$ we mean a pair $(X, f)$, where $f: X \rightarrow X$ is a continuous (not necessarily injective) map. The topological entropy of $f$, defined in AKM65, will be denoted by $h(f)$. This is a fundamental quantitative characteristic of a dynamical system. On the other hand, a dynamical system can possess some qualitative dynamical properties, such as transitivity, weak and strong mixing and exactness (see Section 2 for definitions). It is natural to study the connection between these qualitative properties and entropy. The first result in this direction, due to Blokh [Bl82, says that the topological entropy of any transitive dynamical system on the interval is at least $(1 / 2) \log 2$; moreover, he showed that this bound is the best possible. Hence we have $I^{\mathcal{T}}([0,1])=(1 / 2) \log 2$, where $I^{\mathcal{T}}(X)$ is defined by

$$
I^{\mathcal{T}}(X)=\inf \{h(f): f: X \rightarrow X \text { is transitive }\} .
$$

Here, the infimum is attainable since there is a transitive interval map with the entropy $(1 / 2) \log 2$. In 1987, Blokh Bl87] showed that transitivity implies positiveness of entropy for maps on any graph which is not a circle. Later Alsedà, Kolyada, Llibre and Snoha AKLS99 showed that for an $n$-star $S$ it holds that $I^{\mathcal{T}}(S)=(1 / n) \log 2$. For arbitrary tree $T$, Alsedà, Baldwin, Llibre and Misiurewicz ABLM97. showed that $I^{\mathcal{T}}(T) \geq(1 / n) \log 2$, where $n$ is the number of end points of the tree. For some classes of trees this bound is the best possible (see $\mathrm{Ye00}$ and Ba01) but in general it is still an open problem to determine the value of $I^{\mathcal{T}}(T)$ for arbitrary tree $T$. By Alsedà, del Río and Rodríguez [ARR99], for any graph $X$ which is not a tree it holds that $I^{\mathcal{T}}(X)=0$; they even showed that $I^{\mathcal{E D}}(X)=0$, where

$$
I^{\mathcal{E D}}(X)=\inf \{h(f): f: X \rightarrow X \text { is exactly Devaney chaotic }\} .
$$

By [HKO11, Lemma 8.5], every tree admitting a transitive $P$-linear Markov map admits, for every $\varepsilon>0$, also an exact $P^{\prime}$-linear Markov map $f^{\prime}$ with $h\left(f^{\prime}\right)<h(f)+\varepsilon$. Thus, for trees $T$, the upper bounds for $I^{\mathcal{T}}(T)$ from Ye00 are true also for $I^{\mathcal{E D}}(T)$;

2010 Mathematics Subject Classification. Primary 37B05, 37B20, 37B40; Secondary 54H20.

Key words and phrases. Topological entropy, transitive system, exact system, dendrite.

The author was supported by the Slovak Research and Development Agency under the contract No. APVV-0134-10 and by the Slovak Grant Agency under the grant VEGA 1/0978/11. 
in particular, $I^{\mathcal{E D}}(T)=(1 / n) \log 2$ for every $T \in \mathcal{T}_{0}$ (for the definitions of Ye's classes $\mathcal{T}_{i}$ of trees, see e.g. Subsection 2.3).

A natural class of compact metric spaces containing all trees is the class of dendrites. Recall that a dendrite is a locally connected continuum containing no simple closed curve. Dendrites possess many properties of trees: they have the fixed point property, they are absolute retracts, they are embeddable in the plane, etc. On the other hand, dendrites can be much more complicated than trees; for example there is an uncountable system of pairwise non-homeomorphic dendrites. Recently, the study of dynamics on dendrites attracted many authors; see for instance II98, Ka98, AEO07, Ba07, Ma09, SWZ10, Na12 and other references therein. If $X$ is a dendrite we denote by $E(X)$ the set of all end points of $X$ (that is, points $x \in X$ such that $X \backslash\{x\}$ is connected) and by $B(X)$ the set of all branch points of $X$ (that is, points $x \in X$ such that $X \backslash\{x\}$ has at least three components). Notice that, for a non-degenerate dendrite $X, B(X)$ is always countable and it is empty if only if $X$ is an arc. Further, the set of end points $E(X)$ is always non-empty and it is finite if and only if $X$ is a tree.

In this paper we study infima $I^{\mathcal{T}}(X), I^{\mathcal{E D}}(X)$ for non-degenerate dendrites which are not trees. Every such dendrite $X$ has infinitely many end points and so, for every $n$, it contains an $n$-star $S_{n}$ or an $n$-comb $C_{n}$ (see Corollary 4). Since $I^{\mathcal{T}}\left(S_{n}\right)=(1 / n) \log 2$ by AKLS99] and $I^{\mathcal{T}}\left(C_{n}\right)=(1 / n) \log 2$ for $n=2^{r}$ by $\mathrm{Ye00}$, the dendrite $X$ contains subtrees admitting transitive maps with arbitrarily small entropy. So it is natural to expect that the answer to the following Baldwin's question is affirmative.

Problem ([Ba1] $)$. Is it true that $I^{\mathcal{T}}(X)=0$ for every non-degenerate dendrite $X$ which is not a tree?

The purpose of the present paper is to give a partial affirmative answer to this question. Roughly speaking, we show that $I^{\mathcal{T}}(X)=I^{\mathcal{E D}}(X)=0$ provided $X$ contains "sufficiently many" free arcs. Recall that an arc is a homeomorphic image of $[0,1]$ and a free arc is an arc $A$ such that every non-end point of $A$ is an interior point of $A$ in the topology of $X$.

Theorem A. If $X$ is a non-degenerate dendrite such that no subtree of it contains all free arcs of $X$ then $X$ admits a transitive, even exactly Devaney chaotic map with arbitrarily small entropy. Hence

$$
I^{\mathcal{T}}(X)=I^{\mathcal{E D}}(X)=0,
$$

where the infima are not attainable.

The class of dendrites satisfying the condition from Theorem $\mathrm{A}$ has not been studied in the literature, but it appears to be natural for our construction. Note that in Lemma 5 we will show that the condition is satisfied e.g. for dendrites which are not trees and which have nowhere dense branch points.

Combining Theorem $\mathrm{A}$ with the main result of $[\mathrm{Sp12b}$ we obtain the following corollary. Recall that a continuum $X$ is completely regular provided the only nowhere dense subcontinua of $X$ are degenerate. Since graphs are completely regular, the corollary generalizes [ARR99, Theorem 4.1].

Corollary B. If $X$ is a non-degenerate completely regular continuum which is not a tree then $I^{\mathcal{T}}(X)=I^{\mathcal{E D}}(X)=0$. 
As a by-product, by combining our results with the constructions from Ye00 we obtain the following upper estimate of $I^{\mathcal{E D}}(X)$ for dendrites $X$ based on the number of end points of special subtrees of $X$. Here we say that an edge of a tree $T$ is terminal if it contains an end point of $T$. For the definition of Ye's class $\mathcal{T}_{0}$, see the end of Subsection 2.3 .

Proposition C. If a dendrite $X$ contains a tree $T$ from Ye's class $\mathcal{T}_{0}$ with $n$ end points and with all terminal edges having non-empty interiors in $X$, then

$$
I^{\mathcal{T}}(X) \leq I^{\mathcal{E D}}(X) \leq \frac{\log 2}{n} .
$$

In particular, if $X$ contains a free arc then $I^{\mathcal{E D}}(X) \leq(1 / 2) \log 2$.

The paper is organized as follows. In the next section we summarize all the definitions and facts which will be needed later and we also prove some elementary facts about dendrites. In Section 3 we introduce the so-called $(P, S)$-linear Markov maps on trees and we show how they can be used to define exactly Devaney chaotic tree maps with small entropy. In Section 4 we prove the main results of the paper. Finally, in two appendices we give simple constructions of zero-entropy $(P, S)$-linear Markov maps on stars and combs, and we show, for trees $T$, an upper bound for $I^{\mathcal{E D}}(T)$ which depends only on the number of end points of $T$.

\section{Preliminaries}

In this section we recall basic notations and definitions which are used in the paper. The cardinality of a set $A$ is denoted by $\# A$. By $\mathbb{Z}\left(\mathbb{N}, \mathbb{N}_{0}\right)$ we denote the set of all (positive, non-negative) integers, by $\mathbb{R}$ we denote the set of all reals and by $I$ we denote the unit interval $[0,1]$. For an interval $J,|J|$ is the length (that is, the Lebesgue measure) of it. The symbol $\sqcup$ denotes a disjoint union.

A space is any topological space; it is degenerate if it consists of one point, otherwise it is non-degenerate. A Cantor space is a totally disconnected compact metric space without isolated points. A set is said to be regular closed if it is the closure of its interior or, equivalently, if it is the closure of an open set.

A dynamical system is a pair $(X, f)$, where $X$ is a compact metric space and $f: X \rightarrow X$ is a continuous selfmap of $X$. For non-negative integers $n$ we define the $n$-th iterate $f^{n}$ of $f$ recursively as follows: $f^{0}$ is the identity map on $X$ and $f^{n}=f \circ f^{n-1}$ for any $n \in \mathbb{N}$. A subset $M$ of $X$ is called $f$-invariant (strongly $f$-invariant) provided $f(M) \subseteq M(f(M)=M)$. A subsystem of $(X, f)$ is any dynamical system $\left(M,\left.f\right|_{M}\right)$ where $M$ is a non-empty closed $f$-invariant subset of $X$. If $(X, f)$ is a dynamical system, a point $x$ of $X$ is called fixed if $f(x)=x$ and periodic if $f^{p}(x)=x$ for some $p \in \mathbb{N}$.

The topological entropy of a system $(X, f)$ will be denoted by $h(f)$. A system $(X, f)$ is called transitive if for every non-empty open sets $U, V$ there is $n \in \mathbb{N}$ with $f^{n}(U) \cap V \neq \emptyset$. It is exact if for every non-empty open set $U$ there is $n \in \mathbb{N}$ with $f^{n}(U)=X$. A system $(X, f)$ is (exactly) Devaney chaotic if $X$ is infinite and $f$ is transitive (exact) and has dense periodic points.

2.1. Perron-Frobenius theory. Let $M=\left(m_{i j}\right)_{i j=1}^{n}$ be an $n \times n$ matrix with real entries. A sequence $\pi=\left(p_{j}\right)_{j=0}^{k}(k \geq 1)$ of elements of $\{1, \ldots, n\}$ is called a path if its width $w(\pi)=\prod_{j=1}^{k} m_{p_{j-1} p_{j}}$ is non-zero; the length of $\pi$ is $|\pi|=k$. A path $\left(p_{j}\right)_{j=0}^{k}$ is called a loop if $p_{0}=p_{k}$. The matrix $M$ is called irreducible if for 
every $i, j$ there is a path from $i$ to $j$. If $M$ is irreducible and the greatest common divisor of lengths of all loops is equal to $1, M$ is said to be primitive.

By Perron-Frobenius theorem (see e.g. [Se81, Theorems 1.1 and 1.5]), every nonnegative irreducible matrix $M$ has an eigenvalue $\lambda_{M}>0$ of multiplicity one such that $\lambda_{M} \geq|\lambda|\left(\lambda_{M}>|\lambda|\right.$ if $M$ is primitive) for every eigenvalue $\lambda \neq \lambda_{M}$ of $M$; this $\lambda_{M}$ is called the Perron eigenvalue of $M$. Moreover, there are left and right eigenvectors of $M$ associated with $\lambda_{M}$ which are strictly positive. If $M$ is a general (that is, not necessarily irreducible) non-negative matrix, the spectral radius $\lambda_{M}$ of it (which is equal to the maximal non-negative eigenvalue of $M$ ) will be also called the Perron eigenvalue of $M$. Notice that if $M, N$ are non-negative matrices and $M \leq N$ (that is, $m_{i j} \leq n_{i j}$ for every $i, j$ ), then $\lambda_{M} \leq \lambda_{N}$.

In the special case when $M$ is an irreducible 01-matrix, we have $\lambda_{M} \geq 1$; moreover, $\lambda_{M}=1$ if and only if $M$ is a permutation matrix (that is, it has exactly one entry 1 in each row and each column and 0's elsewhere), see e.g. [Se81, p. 8]. In particular, if $M$ is a primitive 01-matrix, then $\lambda_{M}>1$.

The so-called rome method for calculating the characteristic polynomial of square matrices was proposed in BGMY80, p. 20-21]. Let $M=\left(m_{i j}\right)_{i j=1}^{n}$ be an $n \times n$ matrix. A non-empty subset $\mathcal{R} \subseteq\{1, \ldots, n\}$ is called a rome if for every loop $\left(p_{j}\right)_{j=0}^{k}$ there is $j$ with $p_{j} \in \mathcal{R}$. Let $\mathcal{R}=\left\{r_{1}, \ldots, r_{k}\right\}\left(r_{i} \neq r_{j}\right.$ for $\left.i \neq j\right)$ be a rome. A path $\left(p_{j}\right)_{j=0}^{k}$ is said to be simple (or $\mathcal{R}$-simple) if $p_{0}, p_{k} \in \mathcal{R}$ and $p_{j} \notin \mathcal{R}$ for $1 \leq j<k$. Define a matrix function $R_{\mathcal{R}}$ by

$$
R_{\mathcal{R}}=\left(r_{i j}\right)_{i j=1}^{k}, \quad r_{i j}(x)=\sum_{\pi} w(\pi) x^{-|\pi|} \quad(x \in \mathbb{R}),
$$

where the summation is over all simple paths $\pi$ from $r_{i}$ to $r_{j}$. Let $E$ denote the unit matrix (of an appropriate size). By [BGMY80, Theorem 1.7], the characteristic polynomial $\chi_{M}(x)=\operatorname{det}(M-x E)$ of $M$ satisfies

$$
\chi_{M}(x)=(-1)^{n-k} x^{n} \cdot \operatorname{det}\left(R_{\mathcal{R}}(x)-E\right) \quad \text { for every rome } \mathcal{R} \text { and } x \in \mathbb{R} .
$$

Thus, if $M$ is non-negative with positive spectral radius $\lambda_{M}$, then $\lambda_{M}$ is equal to the maximal positive root of $\operatorname{det}\left(R_{\mathcal{R}}(x)-E\right)$.

2.2. Continua. A continuum is any connected compactum. An arc is a homeomorphic image of the unit interval $I$; the images of 0,1 are called the end points of the arc. An arc $A$ with end points $\{a, b\}$ in a space $X$ is free if $A \backslash\{a, b\}$ is open in $X$. A free $\operatorname{arc} A$ in a connected space $X$ is said to disconnect $X$ if $X \backslash\{x\}$ is not connected for every non-end point $x$ of $A$. A circle or a simple closed curve is a homeomorphic image of the unit circle $\mathbb{S}^{1}$.

Let $X$ be a continuum. A metric $d$ on $X$ is said to be convex provided for every distinct $x, y \in X$ there is $z \in X$ such that $d(x, z)=d(z, y)=d(x, y) / 2$. By Bi49, Theorem 8] every locally connected continuum admits a compatible convex metric. If $X$ is endowed with a convex metric $d$, then for every $a \neq b$ there is an $\operatorname{arc} A$ with end points $a, b$, the length $\mathcal{H}_{d}^{1}(A)$ of which is equal to $d(a, b)$; every such arc will be called geodesic.

2.3. Trees. A graph is a continuum which can be written as the union of finitely many arcs, any two of which are either disjoint or intersect only in one or both of their end points. A tree is a graph containing no simple closed curve. We assume that every tree has associated with it finitely many points of it, called vertices, in such a way that every end point as well as every branch point is a vertex (so we 
allow also vertices of order 2). The edges of a tree are the free arcs both end points of which are vertices. An edge $E$ of a tree $T$ is said to be terminal provided it contains an end point of $T$.

Let $n \geq 3$. Then a tree $T$ is an $n$-star if $T$ has exactly one branch point and this branch point has order $n$. Further, a tree $T$ is an $n$-comb if it has exactly $n-2$ branch points, every branch point has the order 3 and all the branch points belong to an arc. We will also say that a tree $T$ is a 2 -star or a 2 -comb if it is an arc. Notice that $n$-stars and $n$-combs have exactly $n$ end points and that every subtree of a comb (star) is a comb (star).

The following simple lemma will be used later.

Lemma 1. Let $k \geq 3$ and $p \geq 0$ be integers. Let $T$ be a tree with at least $k^{p}$ branch points and such that every branch point of $T$ has the order at most $k$. Then $T$ contains a $(p+3)$-comb.

Proof. We prove the lemma by induction. For $p=0$ it is trivial. Assume now that the assertion of the lemma holds for some $p \geq 0$; we are going to prove that it holds also for $p+1$. Let $T$ be any tree with at least $k^{p+1}$ branch points and such that the order of every branch point is at most $k$. Take an end point $e$ of $X$ and let $b$ be the branch point of $X$ nearest to $e$; then $[b, e]$ is a free arc. The open set $X \backslash\{b\}$ has at most $k$ components the closures of which are trees with the total number of branch points at least $k^{p+1}-1$. Hence there is a component $U$ of $X \backslash\{b\}$ the closure of which has at least $k^{p}$ branch points. Since every branch point of $\bar{U}$ has the order at most $k$, by the induction hypothesis $\bar{U}$ contains a $(p+3)$-comb $S$; we may assume that $b$ is an end point of $S$. Let $e^{\prime}$ be an end point of $X$ contained in a component of $X \backslash\{b\}$ different from both $U$ and $(b, e]$. Then $S \cup[b, e] \cup\left[b, e^{\prime}\right]$ is a $(p+4)$-comb.

In the following we recall Ye's classification of trees YYe0, p. 298-299]. Let $T_{0}, \ldots, T_{n}$ be trees, let $e_{1}, \ldots, e_{n}$ be the end points of $T_{0}$ and $e_{j}^{\prime}$ be a non-end point of $T_{j}(j=1, \ldots, n)$. We say that a tree $T$ is an extension of $T_{1}, \ldots, T_{n}$ over $T_{0}$ if $T$ is homeomorphic to the free union $\bigoplus_{j=0}^{n} T_{j}$ with the pairs $\left(e_{j}, e_{j}^{\prime}\right)(j=1, \ldots, n)$ identified.

Let $r \geq 1, n_{1}, \ldots, n_{r} \geq 2$ be integers and let $T$ be a tree. We say that $T$ belongs to $\mathcal{T}_{0}^{n_{1}, \ldots, n_{r}}$ if either $T$ is an $n_{1}$-star (if $r=1$ ) or $T$ is an extension of some $T_{1}, \ldots, T_{n_{r}} \in \mathcal{T}_{0}^{n_{1}, \ldots, n_{r-1}}$ over an $n_{r}$-star (if $r \geq 2$ ). If $i>0$ we say that $T$ belongs to $\mathcal{T}_{i}^{n_{1}, \ldots, n_{r}}$ if $T$ is a free union of some $T^{\prime} \in \mathcal{T}_{i-1}^{n_{1}, \ldots, n_{r}}$ with an arc $A$, where a non-end point of $T^{\prime}$ is identified with an end point of $A$. Notice that a tree $T$ from $\mathcal{T}_{i}^{n_{1}, \ldots, n_{r}}$ has $n=i+\prod_{l=1}^{r} n_{l}$ end points.

Denote by $\mathcal{T}_{0}$ the union of all $\mathcal{T}_{0}^{n_{1}, \ldots, n_{r}}$ and, for $i \geq 1$, define inductively $\mathcal{T}_{i}$ as the union of all $\mathcal{T}_{i}^{n_{1}, \ldots, n_{r}}$ minus $\bigcup_{0 \leq j<i} \mathcal{T}_{j}$. Notice that every $n$-star as well as every $2^{r}$-comb $(r \geq 1)$ belongs to $\mathcal{T}_{0}$; indeed, for $n$-stars choose $r=1, n_{1}=n$ and for $2^{r}$-combs put $n_{1}=\cdots=n_{r}=2$.

2.4. Dendrites. A dendrite is a (possibly degenerate) locally connected continuum containing no simple closed curve. If $X$ is a dendrite, a point $x \in X$ is called an end (ordinary, branch) point of $X$ if $X \backslash\{x\}$ has one (two, at least three) connected components. The sets of all end, ordinary and branch points of $X$ are denoted by $E(X), O(X)$ and $B(X)$, respectively. A dendrite is a tree if and only if it is non-degenerate and the set of end points is finite. 
Notice that dendrites are uniquely arcwise connected, that is, for every $a \neq b$ there is a unique arc with end points $a, b$. If $X$ is a dendrite and $a, b$ are different points of $X$, the arc with end points $a$ and $b$ will be denoted by $[a, b]$. The set $[a, b] \backslash\{a, b\}$ will be denoted by $(a, b)$; analogously we define $(a, b]$ and $[a, b)$.

Let $X$ be a dendrite and $Y$ be a subcontinuum of $X$. The first point retraction $r=r_{Y}: X \rightarrow Y$ is the map defined by $r(y)=y$ for $y \in Y$ and $r(x)=y_{x}$ for $x \in X \backslash Y$, where $y_{x}$ is the unique point of the boundary of the component of $X \backslash Y$ containing $x$; this map is well defined and continuous, see e.g. [Na92, Lemma 10.25].

The following two lemmas are simple statements about dendrites. We prove just the second one since the first one is trivial.

Lemma 2. If $X$ is a non-degenerate dendrite with finitely many branch points then either $X$ is a tree or $X$ contains an $\omega$-star $S$ which is a union of free arcs.

Lemma 3. If $X$ is a dendrite with infinitely many branch points then at least one of the following holds:

(a) $X$ contains an arc containing infinitely many branch points;

(b) $X$ contains an $\omega$-star which is a union of free arcs.

Proof. Since $B(X)$ is infinite there is a sequence of branch points $\left(b_{n}\right)_{n}$ converging to a point $b \notin\left\{b_{n}: n \in \mathbb{N}\right\}$ (notice that $b$ need not be a branch point). Assume now that there is a component $U$ of the set $X \backslash\{b\}$ containing infinitely many $b_{n}$ 's; without loss of generality we may assume that $b_{n} \in U$ for every $n$. Define the dendrite $Y$ to be the closure of $U$ (that is, $Y=U \cup\{b\}$ ) and take any end point $a$ of $Y$ different from $b$; we show that the arc $A=[a, b]$ contains infinitely many branch points. Indeed, let $r=r_{A}: Y \rightarrow A$ be the first point retraction of $Y$ onto $A$. Since $r$ is continuous, the points $b_{n}^{\prime}=r\left(b_{n}\right) \in A$ converge to $r(b)=b$. Notice that every $b_{n}^{\prime}$ is a branch point of $Y$, hence is a branch point of $X$. This is immediate if $b_{n} \in A$; otherwise $b_{n} \notin A$, so $b_{n}^{\prime} \neq b_{n}$ and $b_{n}^{\prime}$ is not an end point of $A$ (since $b_{n}^{\prime}$ is not an end point of $Y$ ), thus $a, b, b_{n}$ belong to different components of $Y \backslash\left\{b_{n}^{\prime}\right\}$. We also have that $b_{n}^{\prime} \neq b$ for every $n$ due to the fact that $b$ is an end point of $Y$. Now using the fact that $b_{n}^{\prime} \rightarrow b$ we obtain that $\left\{b_{n}^{\prime}: n \in \mathbb{N}\right\}$ is an infinite set of branch points belonging to the $\operatorname{arc} A$.

If every component of $X \backslash\{b\}$ contains only finitely many branch points of $X$ then we have two facts. First, $X \backslash\{b\}$ has infinitely many components $U_{0}, U_{1}, \ldots$ Second, every component $U_{i}$ contains a point $b_{i}$ such that $\left[b, b_{i}\right]$ is a free arc in $\overline{U_{i}}$, hence it is a free $\operatorname{arc}$ in $X$. So in this case we have that $X$ contains an $\omega$-star $\bigcup_{i=0}^{\infty}\left[b, b_{i}\right]$ with every $\left[b, b_{i}\right]$ being a free arc.

Corollary 4. Let $X$ be a non-degenerate dendrite which is not a tree. Then for every $n$ at least one of the following holds:

(a) $X$ contains an $n$-comb;

(b) $X$ contains an $n$-star which is a union of free arcs.

Lemma 5. For a non-degenerate dendrite $X$ the following are equivalent:

(a) no subtree $T$ of $X$ contains all free arcs of $X$;

(b) for every $n \geq 2$ there is a subtree $T$ of $X$ with $n$ end points, the terminal edges of which have non-empty interiors in $X$;

(c) for every $n \geq 2$ there is an $n$-star or an $n$-comb $T$ in $X$, the terminal edges of which have non-empty interiors in $X$. 
Moreover, the properties (e), (f) are equivalent, they imply (d) and, if $X$ is not a tree, (d) implies (a)-(c), where

(d) $B(X)$ is nowhere dense (that is, free arcs are dense in $X$ );

(e) every arc in $X$ has non-empty interior;

(f) $X$ is completely regular.

Notice that, in the assertions (b) and (c), instead of the statement "the terminal edges of which have non-empty interiors in $X$ " we can write "the terminal edges of which are free arcs in $X "$ (recall that in trees we allow vertices of order 2). Notice also that (d) is not equivalent to (e)-(f); e.g. any dendrite such that all branch points of it belong to an arc $A$ and are dense in $A$, satisfies (d) but not (e)-(f).

Proof. For the purpose of the proof let $\mathcal{S}$ denote the system of all subtrees $T$ of $X$ such that every terminal edge of $T$ has non-empty interior in $X$.

(c) implies (b) is immediate. Also (b) implies (a) is trivial; to show this assume, on the contrary, that (b) holds and there is a tree $T$ containing all free $\operatorname{arcs}$ of $X$. Fix any $n>\# E(T)$ and let $T_{n}$ be a subtree of $X$ with $n$ end points such that the terminal edges of $T_{n}$ are free $\operatorname{arcs}$ in $X$. Then $T$ contains every end point of $T_{n}$ and, being uniquely arcwise connected, it has at least $n$ end points - a contradiction.

The fact that (b) implies (c) follows from Lemma 1 In fact, for $n=2,3$ the assertion is trivial, so let $n \geq 4$ and let $T \in \mathcal{S}$ be a tree with $m \geq(n-1)^{n-1}$ end points. If $T$ has a vertex of order at least $n$ then it contains an $n$-star $S$; we may assume that $E(S) \subseteq E(T)$. Then $S \in \mathcal{S}$. Otherwise by Lemma 1 we have that $T$ contains an $(n+2)$-comb $S^{\prime}$; we may again assume that $E\left(S^{\prime}\right) \subseteq E(T)$ and so $S^{\prime} \in \mathcal{S}$.

Now we prove that (a) implies (b). For a subtree $T$ of $X$ let $F_{T}$ denotes the union of all free $\operatorname{arcs} A \subseteq T$. Assume that (a) is true; to show (b) it suffices to prove that the number of end points of trees $T \in \mathcal{S}$ is not bounded from above. Assume, on the contrary, that $n=\sup _{T \in \mathcal{S}} \# E(T)<\infty$. Take $T \in \mathcal{S}$ with $n=\# E(T)$; we may assume that $E(T) \subseteq E(X)$. Let $S$ be the minimal subtree of $T$ containing $F_{T}$; then $\# E(S)=n$ and $S \in \mathcal{S}$. Denote by $x_{1}, \ldots, x_{n}$ the end points of $S$. Let $\left(V_{k}\right)_{k \in K}$ be the (countable) system of (open) components of $X \backslash E(S)$ disjoint from $S$ and let $x_{m_{k}}$ be the boundary point of $V_{k}(k \in K)$. Let $K^{\prime} \subseteq K$ be the set of all $k$ 's such that $Y_{k}=\bar{V}_{k}$ has a free arc. Since, by (a), $S$ does not contain all free arcs of $X$, the set $K^{\prime}$ is non-empty. Moreover, if $k \in K^{\prime}$ then $x_{m_{k}} \in E(S)$ and if $k \neq k^{\prime}$ belong to $K^{\prime}$ then $x_{m_{k}} \neq x_{m_{k^{\prime}}}$ since otherwise we can easily construct a tree $S^{\prime} \supseteq S$ from $\mathcal{S}$ with than $(n+1)$ end points. An analogous argument shows that for every $k \in K^{\prime}$ there is an arc $A_{k}$ with $x_{m_{k}} \in E\left(A_{k}\right)$ containing all free arcs of $Y_{k}$. But then $S^{\prime}=S \cup \bigcup_{k \in K^{\prime}} A_{k}$ is a subtree of $X$ (use that $K^{\prime}$ is finite) containing all free $\operatorname{arcs}$ of $X-$ a contradiction.

To show that (e) implies (d) assume that $B(X)$ is not nowhere dense. Take a nonempty connected open subset $V \subseteq \overline{B(X)}$ and put $Y=\bar{V}$. Since $B(Y)=B(X) \cap V$ and $B(X)$ is dense in $V$ we have that $\overline{B(Y)}=Y$. Hence any arc $A \subseteq Y$ has empty interior in $Y$ and so it has empty interior in $X$.

The fact that (f) implies (e) is immediate since every arc $A \subseteq X$ is a nondegenerate subcontinuum of $X$. The condition (e) implies (f) since if $Y$ is a nondegenerate nowhere dense subcontinuum of $X$ then it contains a nowhere dense arc $A \subseteq Y$. Finally, if $X$ is not a tree then (d) implies (a), since if branch points are 
nowhere dense then free arcs are dense in $X$; hence the only subcontinuum of $X$ containing all free $\operatorname{arcs}$ of $X$ is $X$ itself.

2.5. Totally regular continua. A continuum $X$ is totally regular if for every $x \in X$ and every countable set $P \subseteq X$ there is a basis of neighborhoods of $x$ with finite boundary not intersecting $P$. Every dendrite, as well as every completely regular continuum (that is, continuum containing no non-degenerate nowhere dense subcontinuum) is totally regular. Totally regular continua are locally connected one-dimensional continua. By [EH43, every totally regular continuum admits a compatible convex metric $d$ such that $(X, d)$ has finite one-dimensional Hausdorff measure $\mathcal{H}_{d}^{1}(X)$.

2.6. Length-expanding Lipschitz maps. Here we prove a slight refinement of the main result of Sp12a valid for dendrites. First we recall the definition of lengthexpanding Lipschitz maps. If $X$ is a non-degenerate totally regular continuum and $\mathcal{C}$ is a family of non-degenerate subcontinua of $X$, we say that $\mathcal{C}$ is dense if every non-empty open set in $X$ contains a member of $\mathcal{C}$. By $\mathcal{C}_{I}$ we denote the system of all non-degenerate closed subintervals of $I$; the Euclidean metric on $I$ is denoted by $d_{I}$. A map $f$ is called Lipschitz- $L$ if $\operatorname{Lip}(f) \leq L$.

Definition 6 ([Sp12a $)$. Let $X=(X, d), X^{\prime}=\left(X^{\prime}, d^{\prime}\right)$ be non-degenerate (totally regular) continua of finite length and let $\mathcal{C}, \mathcal{C}^{\prime}$ be dense systems of subcontinua of $X, X^{\prime}$, respectively. We say that a continuous map $f: X \rightarrow X^{\prime}$ is length-expanding with respect to $\mathcal{C}, \mathcal{C}^{\prime}$ if there exists $\varrho>1$ such that for every $C \in \mathcal{C}$ we have $f(C) \in \mathcal{C}^{\prime}$ and

$$
\text { if } \quad f(C) \neq X^{\prime} \quad \text { then } \quad \mathcal{H}_{d^{\prime}}^{1}(f(C)) \geq \varrho \cdot \mathcal{H}_{d}^{1}(C) .
$$

Moreover, if $f$ is surjective and Lipschitz- $L$ we say that $f:(X, d, \mathcal{C}) \rightarrow\left(X^{\prime}, d^{\prime}, \mathcal{C}^{\prime}\right)$ is $(\varrho, L)$-length-expanding Lipschitz. Sometimes we briefly say that $f$ is $(\varrho, L)$-LEL or only $L E L$.

In this paper we will use the above notions also in the case when $\varrho \in(0,1]$; to distinguish them, in such a case we will say that $f$ is length-expanding* or $(\varrho, L)$ $L E L^{*}$.

Let $X$ be a non-degenerate dendrite. By e.g. [Na92, Theorem 10.27], we may write $X$ in the form

$$
\begin{aligned}
& X=\text { closure }\left(\bigcup_{n \in N} X_{n}\right), \quad \text { where } X_{n}=A_{1} \cup \cdots \cup A_{n}, \\
& A_{n}=\left[a_{n}, b_{n}\right] \text { are arcs with } A_{n} \cap X_{n-1}=\left\{a_{n}\right\} \text { for } n \geq 2
\end{aligned}
$$

(here $N=\mathbb{N}$ provided $X \neq A_{1}$ and $N=\{1\}$ otherwise). Define a linear ordering on every $A_{n}$ in such a way that $a_{n}<b_{n}$. We will need the following refinement of Sp12a, Theorem C]. Till the end of the paper we fix a constant $q \in(0,1 / 3]$.

Proposition 7. Let $X$ be a dendrite and $a \neq b$ be points of it. Write $X$ in the form (2.2) with $a_{1}=a, b_{1}=b$ and fix convex metrics $d_{n}$ on $A_{n}(n \in \mathbb{N})$. Then there are a convex metric $d=d_{X, a, b}$ on $X$ and Lipschitz surjections $\varphi=\varphi_{X, a, b}: I \rightarrow X$, $\psi=\psi_{X, a, b}: X \rightarrow I$ with the following properties:

(a) $\mathcal{H}_{d}^{1}(X)=1, d(a, b)>1-q$; 
(b) the system

$\mathcal{C}=\mathcal{C}_{X, a, b}=\{\varphi(J): J$ is a non-degenerate closed subinterval of $I\}$

of subcontinua of $X$ is dense;

(c) the maps

$$
\varphi:\left(I, d_{I}, \mathcal{C}_{I}\right) \rightarrow(X, d, \mathcal{C}) \quad \text { and } \quad \psi:(X, d, \mathcal{C}) \rightarrow\left(I, d_{I}, \mathcal{C}_{I}\right)
$$

are $(\varrho, L)-L E L^{*}$, where $0<\varrho<1<L$ are constants depending only on $q$, and $\varphi(0)=a, \varphi(1)=b, \psi(a)=0, \psi(b)=1$;

(d) there is $c>0$ such that $\psi(x)=c \cdot d(a, x)$ for every $x \in X$;

(e) for every $n$ there is $c_{n}>0$ such that $d(x, y)=c_{n} \cdot d_{n}(x, y)$ whenever $x, y \in A_{n}$;

(f) $\mathcal{H}_{d}^{1}\left(A_{n+1}\right) \leq q \cdot \mathcal{H}_{d}^{1}\left(A_{n}\right)$ for every $n$;

(g) if $r=r_{A_{1}}: X \rightarrow A_{1}$ is the first point retraction of $X$ onto $A_{1}$, then

$$
r \circ \varphi(s) \leq r \circ \varphi(t) \quad \text { for every } s \leq t \text { from } I \text {. }
$$

Notice that, if $X=[a, b]$ is an arc, then $\mathcal{C}_{X, a, b}$ is the system of all subarcs of $X$.

Proof. If $X=[a, b]$ the statement is trivial; so assume that $N=\mathbb{N}$. For every $n$ let $f_{n}: X_{n+1} \rightarrow X_{n}$ be the first point retraction (that is, $f_{n}(x)=x$ for $x \in X_{n}$ and $f_{n}(x)=a_{n+1}$ for $x \in A_{n+1}$ ). By Anderson-Choquet embedding theorem (see e.g. [Na92, 2.10]), $X$ is homeomorphic to the inverse $\operatorname{limit} \underset{\lim }{\longleftarrow}\left(X_{n}, f_{n}\right)$. Repeating the construction from [Sp12a, Section 5.2] (see also the remark after Sp12a, Corollary 26]) word-by-word, with $\tilde{d}_{n}=c_{n} \cdot d_{n}$ where $c_{n}$ 's are sufficiently small, we obtain $d_{X, a, b}, \varphi_{X, a, b}$ and $\psi_{X, a, b}$ such that all the properties (a)-(g) are satisfied.

The following can be proved in the same way as $[\mathrm{Sp12 \textrm {a }}$, Theorem D]. For $k \in \mathbb{N}$, let $f_{k}: I \rightarrow I$ denote the continuous map which fixes 0 and maps every $[(i-$ $1) / k, i / k]$ linearly onto $I$.

Proposition 8. Keeping the notation from Proposition 7 , for every $\rho>1$, every non-degenerate totally regular continua $X, X^{\prime}$ and every pairs of distinct points $a, b \in X, a^{\prime}, b^{\prime} \in X^{\prime}$ there are a constant $L_{\rho}$ (depending only on $\rho$ ) and $a\left(\rho, L_{\rho}\right)$ LEL map

$$
f:\left(X, d_{X, a, b}, \mathcal{C}_{X, a, b}\right) \rightarrow\left(X^{\prime}, d_{X^{\prime}, a^{\prime}, b^{\prime}}, \mathcal{C}_{X^{\prime}, a^{\prime}, b^{\prime}}\right)
$$

with $f(a)=a^{\prime}$ and $f(b)=b^{\prime}$. Moreover, $f$ is equal to the composition $\varphi_{X^{\prime}, a^{\prime}, b^{\prime}} \circ$ $f_{k} \circ \psi_{X, a, b}$ with some odd $k \geq 3$.

Finally, we prove the next lemma which is a consequence of Proposition 7

Lemma 9. Keeping the notation from Proposition 7 , there is a constant $\gamma>0$ with the following property: For every dendrite $X$ and every two distinct points $a, b$ of it, there exists a

$$
(\gamma, 1)-L E L^{*} \text { retraction } r=r_{X, a, b}:\left(X, d_{X, a, b}, \mathcal{C}_{X, a, b}\right) \rightarrow\left(A, d_{A}, \mathcal{C}_{A}\right),
$$

where $A=[a, b], d_{A}$ is the restriction of $d_{X, a, b}$ onto $A \times A$ and $\mathcal{C}_{A}$ is the system of all subarcs of $A$.

Proof. Let $\varrho$ be the constant from (c) of Proposition 7 and put $\gamma=(1-q)^{2} \varrho / 4$; we prove that this $\gamma$ satisfies the above stated property. To this end, let $X$ be a dendrite, $a \neq b$ be points of $X$ and $A=[a, b]$. Write $X$ in the form (2.2) with $A_{1}=A$. If $X=A$, the assertion is trivial (take $r=\mathrm{id}_{X}$ ); so assume that 
$X \neq A$. Proposition 7 gives us a convex metric $d=d_{X, a, b}$ and maps $\varphi_{X, a, b}, \psi_{X, a, b}$ satisfying the conditions (a)-(g); put $\mathcal{C}=\mathcal{C}_{X, a, b}$ and let $r_{A}: X \rightarrow A$ be the first point retraction. Let $y_{k}(k \in K)$ be all points $y$ of $A$ with non-degenerate preimage $r_{A}^{-1}(y)$. Put $Y_{k}=r_{A}^{-1}\left(y_{k}\right)(k \in K)$; then $Y_{k}$ 's are pairwise disjoint non-degenerate subdendrites of $X$ and $X=A \cup \bigcup_{k} Y_{k}$ Put $t_{0}=d(a, b), t_{k}=d\left(a, y_{k}\right)(k \geq 1)$ and for any $t \in\left[0, t_{0}\right]$ denote by $a_{t}$ the unique point of $A$ satisfying $d\left(a_{t}, a\right)=t$; hence e.g. $a_{t_{k}}=y_{k}$ and $\mathcal{H}_{d}^{1}\left(\left[a_{s}, a_{t}\right]\right)=t-s$ for any $s<t$. Now define $r=r_{X, a, b}: X \rightarrow A$ by

$$
r(x)= \begin{cases}x & \text { if } x \in A \\ a_{t_{k}+d\left(x, y_{k}\right)} & \text { if } x \in Y_{k} \text { with } t_{k} \leq t_{0} / 2 \\ a_{t_{k}-d\left(x, y_{k}\right)} & \text { if } x \in Y_{k} \text { with } t_{k}>t_{0} / 2 .\end{cases}
$$

By Proposition 7(a) and the choice of $q, t_{0}>2 / 3$ and so $d\left(x, y_{k}\right)<1 / 3<t_{0} / 2$ for every $x \in Y_{k}$. Hence $r(x)$ is well defined for every $x$. Trivially, $r$ is a continuous retraction of $X$ onto $A$. Using convexity of $d$ one can easily show that $\operatorname{Lip}(r)=1$.

It remains to prove that $r$ is $\gamma$-length expanding*. To this end, fix $C \in \mathcal{C}$, $C=\varphi_{X, a, b}\left(\left[s_{0}, s_{1}\right]\right)$ and put $u_{i}=r_{A} \circ \varphi_{X, a, b}\left(s_{i}\right)(i=0,1)$. By Proposition 7 (g) we may write

$$
C=\left[C_{0} \sqcup\left(\bigsqcup_{k: y_{k} \in\left(u_{0}, u_{1}\right)} Y_{k}\right) \sqcup C_{1}\right] \cup\left[u_{0}, u_{1}\right]
$$

with $C_{i}(i=0,1)$ being connected (either degenerate or belonging to $\left.\mathcal{C}\right)$. We will assume that there is $k$ with $y_{k} \in\left(u_{0}, u_{1}\right)$; the other (simpler) case can be described analogously. Put $n_{0}=\min \left\{n: A_{n} \subseteq Y_{k}, u_{0}<y_{k}<u_{1}\right\} \geq 2$. Then, using (f) from Proposition 7

$$
\begin{aligned}
\mathcal{H}_{d}^{1}(C) & =\mathcal{H}_{d}^{1}\left(C_{0}\right)+\sum_{k: y_{k} \in\left(u_{0}, u_{1}\right)} \mathcal{H}_{d}^{1}\left(Y_{k}\right)+\mathcal{H}_{d}^{1}\left(C_{1}\right)+\mathcal{H}_{d}^{1}\left(\left[u_{0}, u_{1}\right]\right) \\
& \leq \frac{4}{1-q} \cdot \max \left\{\mathcal{H}_{d}^{1}\left(C_{0}\right), \mathcal{H}_{d}^{1}\left(A_{n_{0}}\right), \mathcal{H}_{d}^{1}\left(C_{1}\right), \mathcal{H}_{d}^{1}\left(\left[u_{0}, u_{1}\right]\right)\right\}
\end{aligned}
$$

On the other hand, by Proposition 7(d) we have $\psi(x)=c \cdot d(a, x)$ for every $x \in X$, where $c=1 / d(a, b) \in(1,1 /(1-q))$. For given $k$ and a continuum $Y \subseteq Y_{k}$ from $\mathcal{C}$ containing $y_{k}$ we have $\psi(Y)=\left[\psi\left(y_{k}\right), \psi\left(z_{k}\right)\right]$, where $z_{k}$ is such that $d\left(z_{k}, y_{k}\right)=$ $\max _{y \in Y_{k}} d\left(z, y_{k}\right)$. Thus, by convexity of $d,|\psi(Y)|=c \cdot\left(d\left(a, z_{k}\right)-d\left(a, y_{k}\right)\right)=$ $c \cdot d\left(y_{k}, z_{k}\right)=c \cdot \mathcal{H}_{d}^{1}(r(Y))$. By Proposition $7(\mathrm{c})$, for every such continuum $Y$ we have $\mathcal{H}_{d}^{1}(r(Y)) \geq(\varrho / c) \cdot \mathcal{H}_{d}^{1}(Y) \geq \varrho(1-q) \cdot \mathcal{H}_{d}^{1}(Y)$. Hence, using the fact that $\left.r\right|_{A}$ is the identity,

$$
\begin{aligned}
\mathcal{H}_{d}^{1}(r(C)) & \geq \max \left\{\mathcal{H}_{d}^{1}\left(r\left(C_{0}\right)\right), \mathcal{H}_{d}^{1}\left(r\left(A_{n_{0}}\right)\right), \mathcal{H}_{d}^{1}\left(r\left(C_{1}\right)\right), \mathcal{H}_{d}^{1}\left(r\left(\left[u_{0}, u_{1}\right]\right)\right)\right\} \\
& \geq \varrho(1-q) \cdot \max \left\{\mathcal{H}_{d}^{1}\left(C_{0}\right), \mathcal{H}_{d}^{1}\left(A_{n_{0}}\right), \mathcal{H}_{d}^{1}\left(C_{1}\right), \mathcal{H}_{d}^{1}\left(\left[u_{0}, u_{1}\right]\right)\right\} .
\end{aligned}
$$

Thus, by the choice of $\gamma, \mathcal{H}_{d}^{1}(r(C)) \geq \gamma \cdot \mathcal{H}_{d}^{1}(C)$ for every $C \in \mathcal{C}$. The lemma is proved.

2.7. $\quad P$-Lipschitz maps. In Sp12b we introduced the so-called $P$-Lipschitz maps (where $P$ is a finite invariant set) and we gave an upper bound for their entropy [Sp12b, Proposition 3.3]. We recall the definition as well as the corresponding result here. If $X$ is a continuum, a splitting of $X$ is any system $\mathcal{A}=\left\{X_{1}, \ldots, X_{n}\right\}$ of non-degenerate subcontinua covering $X$ such that $P_{\mathcal{A}}=\bigcup_{i \neq j} X_{i} \cap X_{j}$ is finite. 
Definition 10 ([Sp12b] $)$. Let $X$ be a non-degenerate continuum and $f: X \rightarrow X$ be a continuous map. Let $P$ be a finite $f$-invariant subset of $X, \mathcal{A}$ be a splitting of $X$ with $P_{\mathcal{A}} \subseteq P$ and $\left(L_{A}\right)_{A \in \mathcal{A}}$ be positive constants. Then we say that $f$ is $P$-Lipschitz (w.r.t. the splitting $\mathcal{A}$ and the constants $\left(L_{A}\right)_{A \in \mathcal{A}}$ ) if, for every $A \in \mathcal{A}$, $f(A)$ is non-degenerate and $\operatorname{Lip}\left(\left.f\right|_{A}\right) \leq L_{A}$.

Let $f$ be a $P$-Lipschitz map w.r.t. $\mathcal{A}$. For $A, B \in \mathcal{A}$ we write $A \rightarrow B$ or, more precisely, $A \stackrel{f}{\rightarrow} B$ provided $f(A)$ intersects the interior of $B$. The $P$-transition graph $G_{f}$ of $f$ is the directed graph the vertices of which are the sets $A \in \mathcal{A}$ and the edges of which correspond to $A \rightarrow B$. The corresponding 01-transition matrix will be called the $P$-transition matrix and will be denoted by $M_{f}$. For an integer $n \geq 1$ put

$$
\mathcal{A}^{n}=\left\{\mathbb{A}=\left(A_{0}, A_{1}, \ldots, A_{n-1}\right): A_{i} \in \mathcal{A}, A_{0} \rightarrow A_{1} \rightarrow \cdots \rightarrow A_{n-1}\right\} ;
$$

that is, $\mathcal{A}^{n}$ is the set of all paths of length $n-1$ in $G_{f}$. Finally, for non-empty $\mathcal{B} \subseteq \mathcal{A}$ put

$$
\theta_{\mathcal{B}}=\limsup _{n \rightarrow \infty} \frac{k_{n}^{\mathcal{B}}}{n} \quad \text { where } \quad k_{n}^{\mathcal{B}}=\max _{\left(A_{0}, \ldots, A_{n-1}\right) \in \mathcal{A}^{n}} \#\left\{j: A_{j} \notin \mathcal{B}\right\},
$$

which is a quantity measuring the maximal "asymptotic frequency" of occurrences of $A \in \mathcal{A} \backslash \mathcal{B}$ in paths of $f$. The following proposition gives an upper bound for the entropy of $P$-Lipschitz maps. There and below, $\log ^{+} x$ denotes $\max \{\log x, 0\}$.

Proposition $11([\mathrm{Sp} 12 \mathrm{~b}])$. Let $X$ be a non-degenerate totally regular continuum endowed with a convex metric d such that $\mathcal{H}_{d}^{1}(X)<\infty$. Let $f: X \rightarrow X$ be $P$ Lipschitz w.r.t. $\mathcal{A}$ and $\left(L_{A}\right)_{A \in \mathcal{A}}$. Then, for every non-empty subsystem $\mathcal{B}$ of $\mathcal{A}$,

$$
h(f) \leq \log ^{+} L_{\mathcal{B}}+2 \theta_{\mathcal{B}} \log ^{+} L_{\mathcal{A}},
$$

where $L_{\mathcal{B}}=\max _{A \in \mathcal{B}} L_{A}$ and $L_{\mathcal{A}}=\max _{A \in \mathcal{A}} L_{A}$.

\section{MARKOV MAPS ON TREES}

Let $T$ be a tree and $f: T \rightarrow T$ be a continuous map. Assume that a finite set $P \subset T$ is $f$-invariant and contains all vertices of $T$. We say that $f$ is $P$-Markov if it is monotone on each component of $T \backslash P$. The closures of the components of $T \backslash P$ are called $P$-basic arcs of $f$. The $P$-transition graph of $f$ with respect to $P$ is the directed graph having $P$-basic arcs as vertices, with an edge from $A$ to $B$ (we write $A \rightarrow B$ ) if and only if $B \subseteq f(A)$. The corresponding 01-matrix will be denoted by $M_{f}$ and called the $P$-transition matrix. For the following results see e.g. Ba01, Proposition 1.4, Corollary 1.11].

Lemma 12. Let $T$ be a tree and let $f: T \rightarrow T$ be a P-Markov map with the transition matrix $M$ and the Perron-Frobenius value $\lambda_{M}$. Then $h(f)=\log ^{+} \lambda_{M}$.

Lemma 13. Let $T$ be a tree and let $f: T \rightarrow T$ be a P-Markov map with the transition matrix $M$. Then

(a) $f$ is transitive if and only if $M$ is irreducible and not a permutation matrix;

(b) $f$ is exact if and only if $M$ is primitive.

A special class of Markov maps consists of the so-called $P$-linear Markov maps. Let $T$ be a tree endowed with a convex metric $d$. Let $f: T \rightarrow T$ be a $P$-Markov 
map. We say that $f$ is $P$-linear if for every component $C$ of $T \backslash P$ there is a positive constant $\lambda_{C}$ such that

$$
d(f(x), f(y))=\lambda_{C} \cdot d(x, y) \quad \text { for every } x, y \in C .
$$

Moreover, if there is $\lambda$ such that every $\lambda_{C}$ is equal to $\lambda$, we say that $f$ is $P$-linear with constant slope $\lambda$.

By BC01, every $P$-Markov tree map $f: T \rightarrow T$ is semiconjugate to a $P^{\prime}$-linear tree map $f^{\prime}: T^{\prime} \rightarrow T^{\prime}$ with constant slope $\lambda^{\prime}=e^{h(f)}$. The following trivial fact shows that if $f$ is $P$-linear and transitive then the "constant slope" can be achieved by a simple change of the metric on $T$.

Lemma 14. Let $T$ be a tree with a convex metric $d$ and let $f: T \rightarrow T$ be a transitive P-linear Markov map on $T$ with the entropy $h(f)=\log \lambda$. Then there is an equivalent convex metric $d^{\prime}$ on $T$ such that $f:\left(T, d^{\prime}\right) \rightarrow\left(T, d^{\prime}\right)$ is a P-linear Markov map with constant slope $\lambda$.

Proof. Denote the $P$-basic arcs by $A_{i}=\left[a_{i}, b_{i}\right], i=1, \ldots, m$. Since $T$ is uniquely arcwise connected, every $A_{i}$ is geodesic and so its length is $\mathcal{H}_{d}^{1}\left(A_{i}\right)=d\left(a_{i}, b_{i}\right)$. Since $f$ is transitive, its $P$-transition matrix $M$ is irreducible. Thus $\lambda$ is the Perron eigenvalue of $M$ with a strictly positive right eigenvector $\alpha=\left(\alpha_{1}, \ldots, \alpha_{m}\right)$; that is, $M \cdot \alpha=\lambda \alpha$. Let $d^{\prime}$ be the unique convex metric on $T$ such that

$$
d^{\prime}(x, y)=\frac{\alpha_{i}}{d\left(a_{i}, b_{i}\right)} \cdot d(x, y) \quad \text { for every } i \text { and } x, y \in A_{i} .
$$

Trivially $f$ is $P^{\prime}$-linear on $\left(T, d^{\prime}\right)$ and $\mathcal{H}_{d^{\prime}}^{1}\left(A_{i}\right)=\alpha_{i}$ for every $i$. By the choice of $\alpha$ we have that

$$
\lambda \cdot \mathcal{H}_{d^{\prime}}^{1}\left(A_{i}\right)=\sum_{j: A_{i} \rightarrow A_{j}} \mathcal{H}_{d^{\prime}}^{1}\left(A_{j}\right)=\mathcal{H}_{d^{\prime}}^{1}\left(f\left(A_{i}\right)\right)
$$

for every $i$. Hence $f$ has constant slope $\lambda$ on $\left(T, d^{\prime}\right)$.

3.1. ( $P, S)$-linear Markov maps. Here we introduce the so-called $(P, S)$-linear Markov maps, where $S=\left(s_{0}, s_{1}, \ldots, s_{n}\right)$ is a tuple of points from $P$; see Definition 15. Maps with this property can be used to define exactly Devaney chaotic tree maps with "controlled" entropy, see Proposition 19.

Definition 15. Let $T$ be a tree with a convex metric, $P \subseteq T$ be finite and $f: T \rightarrow T$ be a $P$-linear Markov map. Let $n \geq 1$ and $S=\left(s_{0}, s_{1}, \ldots, s_{n}\right)$ be a tuple of distinct points from $P$. We say that $f$ is $(P, S)$-linear if

(a) $f\left(s_{i}\right)=s_{i+1}$ for every $0 \leq i<n$;

(b) $A_{S}=\left[s_{0}, s_{n}\right]$ is a $P$-basic arc and $s_{n}$ is an end point of $T$;

(c) for every $P$-basic arc $A$ different from $A_{S}$ there is a path from $A$ to $A_{S}$.

Tree maps with this property were constructed e.g. in the proof of AKLS99, Theorem 1.2] and also in the proofs of [Ye00, Lemmas 4.6,4.7]. Let us formulate the latter fact as a lemma. (For Ye's classification of trees, see Subsection 2.3.)

Lemma 16. Let $T$ be a tree and let $i$ be such that $T \in \mathcal{T}_{i}$. Then, for every $\varepsilon>0$, there are a finite set $P$, a tuple $S=\left(s_{0}, \ldots, s_{m}\right)$ with $\left\{s_{1}, \ldots, s_{m}\right\} \subseteq E(T)$ and a transitive $(P, S)$-linear map $f: T \rightarrow T$ with

$$
h(f)<\frac{\log 2}{m}+\varepsilon, \quad \text { where } m=\# E(T)-i .
$$


Proof. We will keep the notation from the proofs of [Ye00, Lemmas 4.6, 4.7]. Let $T \in \mathcal{T}_{i}^{n_{1}, \ldots, n_{r}}$, where $i \geq 0, r \geq 1$ and $n_{1}, \ldots, n_{r} \geq 2$. Put $m=m_{0}=\prod_{l=1}^{r} n_{l}=$ $\# E(T)-i$ and $n_{0}=r+i$. The end points of $T$ are $t_{q}^{1}\left(1 \leq q \leq m_{0}\right)$ and $y^{j}$ $(1 \leq j \leq i)$. The maps $f_{n}=f_{i, r, n}$ constructed in the proofs are $P_{n}$-linear Markov maps (we put $P_{n}=Q_{n}$ if $i \geq 1$ ). If we put $S_{n}=\left(s_{0}, \ldots, s_{m_{0}}\right)$ with $s_{q}=t_{q}^{1}$ for $q=1, \ldots, m_{0}$, and $s_{0}=x_{\left(n+n_{0}\right) m_{0}}^{1}$, then the first two conditions from Definition 15] are satisfied, see [Ye00, p. 301]. The third one is satisfied trivially since the maps $f_{n}$ are transitive. Thus every $f_{n}$ is $\left(P_{n}, S_{n}\right)$-linear. Since $\lim h\left(f_{n}\right)=\left(1 / m_{0}\right) \log 2$, the proof is finished.

Let us note that in the proofs of Propositions 19 and 20 we do not need to assume that the considered $(P, S)$-linear maps are transitive. In Appendix 1 we give simple constructions of (non-transitive) zero entropy $(P, S)$-linear Markov maps on stars and combs; this makes the proof of Theorem not dependent on the results of Ye00.

3.2. Exact $P$-linear Markov maps. In Proposition 19 we show that every $(P, S)$-linear Markov map $f$ on a tree $R$ can be used to define an exact map $g$ on a tree $T \supseteq R$ such that the entropy of $g$ is small provided the entropy of $f$ is small and the cardinality of $S$ is large. Such maps $g$ will be used in Section 4 in the construction of exact small entropy maps on dendrites, see Proposition 20, First we introduce some notation which will be used for the entropy estimation.

Let $\mathcal{P}$ denote the system of maps of two variables $x, N$ of the type

$$
\psi:(1, \infty) \times \mathbb{N} \rightarrow \mathbb{R}, \quad \psi(x, N)=\frac{b_{1, N}}{x}+\frac{b_{2, N}}{x^{2}}+\cdots+\frac{b_{i, N}}{x^{i}}+\ldots
$$

such that for some $b=b_{\psi}>0$ and $k=k_{\psi} \in \mathbb{N}$ it holds that

$$
\left|b_{i, N}\right| \leq b \cdot N^{k} \cdot i^{k} \quad \text { for every } i, N \in \mathbb{N} .
$$

Notice that (3.1) guarantees that $\psi(x, N)$ is always finite, since

$$
|\psi(x, N)| \leq b \cdot N^{k} \cdot \nu_{k}(x), \quad \text { where } \quad \nu_{k}(x)=\sum_{i=1}^{\infty} \frac{i^{k}}{x^{i}}<\infty .
$$

Lemma 17. The system $\mathcal{P}$ is closed under multiplication and linear combinations, that is, $c \psi+c^{\prime} \psi^{\prime} \in \mathcal{P}$ and $\psi \cdot \psi^{\prime} \in \mathcal{P}$ for every $\psi, \psi^{\prime} \in \mathcal{P}$ and $c, c^{\prime} \in \mathbb{R}$.

Proof. Let $c, c^{\prime} \in \mathbb{R}, \psi, \psi^{\prime} \in \mathcal{P}$ and let $b_{i, N}, b_{i, N}^{\prime}$ be the coefficients of $\psi, \psi^{\prime}$, respectively. Put $b=b_{\psi}, k=k_{\psi}, b^{\prime}=b_{\psi^{\prime}}$ and $k^{\prime}=k_{\psi^{\prime}}$.

The fact that $c \psi+c^{\prime} \psi^{\prime} \in \mathcal{P}$ is trivial. To show that $\varphi=\psi \cdot \psi^{\prime}$ belongs to $\mathcal{P}$, realize that $\varphi(x, N)=\sum_{i=2}^{\infty} c_{i, N} / x^{i}$, where the coefficients $c_{i, N}$ satisfy

$$
\left|c_{i, N}\right|=\left|\sum_{j=1}^{i-1} b_{j, N} \cdot b_{i-j, N}^{\prime}\right| \leq b \cdot b^{\prime} \cdot N^{k+k^{\prime}} \cdot \sum_{j=1}^{i-1} j^{k} \cdot(i-j)^{k^{\prime}} .
$$

The sum in the right-hand side can be bounded from above by $i^{k+k^{\prime}} \cdot(i-1)<$ $i^{k+k^{\prime}+1}$. So we can take $b_{\varphi}=b \cdot b^{\prime}$ and $k_{\varphi}=k+k^{\prime}+1$.

Denote by $\mathcal{P}_{\text {small }}$ the subsystem of $\mathcal{P}$ consisting of those $\psi \in \mathcal{P}$ for which there is $l \in \mathbb{N}$ (not depending on $N$ ) with $b_{1, N}=b_{2, N}=\cdots=b_{N-l, N}=0$ for every $N \geq l$.

Lemma 18. The following are true: 
(a) $\psi \in \mathcal{P}_{\text {small }}$ if and only if there is $\varphi \in \mathcal{P}$ and $l \in \mathbb{N}$ such that

$$
\psi(x, N)=\frac{1}{x^{N-l}} \cdot \varphi(x, N) .
$$

(b) If $\delta>0$ and $\psi \in \mathcal{P}_{\text {small }}$ then, for $N \rightarrow \infty, x^{N / 2} \cdot \psi(x, N)$ converges to 0 uniformly on $[1+\delta, \infty)$.

(c) $c \psi+c^{\prime} \psi^{\prime} \in \mathcal{P}_{\text {small }}$ and $\psi \cdot \psi^{\prime \prime} \in \mathcal{P}_{\text {small }}$ for every $\psi, \psi^{\prime} \in \mathcal{P}_{\text {small }}, \psi^{\prime \prime} \in \mathcal{P}$ and $c, c^{\prime} \in \mathbb{R}$.

Proof. (a) If $\psi \in \mathcal{P}_{\text {small }}$, there is $l \in \mathbb{N}$ such that $b_{i, N}=0$ for every $N$ and every $i \leq N-l$. Put $\varphi(x, N)=\sum_{i} c_{i, N} x^{-i}$, where $c_{i, N}=b_{N-l+i, N}$. Then $\psi(x, N)=x^{-(N-l)} \varphi(x, N)$ and $\left|c_{i, N}\right| \leq b \cdot N^{k} \cdot(N-l+i)^{k} \leq b \cdot N^{2 k} \cdot i^{k}$, so $\varphi \in \mathcal{P}$. The reverse implication is obvious.

(b) follows from the inequality $\left|x^{N / 2} \cdot \psi(x, N)\right| \leq x^{-(N / 2-l)} \cdot b \cdot N^{k} \cdot \nu_{k}(x)$, where $k=k_{\varphi}, b=b_{\varphi}$ with $\varphi$ is given by (a) and $\nu_{k}(x)=\sum_{i=1}^{\infty} i^{k} x^{-i}$.

(c) follows from (a) and Lemma 17.

Proposition 19. Let $R$ be a tree and let $f: R \rightarrow R$ be a $(P, S)$-linear map, where $S=\left(s_{0}, s_{1}, \ldots, s_{n}\right)$ with $n \geq 2$. Let $T \supseteq R$ be a tree such that

$$
T=R \sqcup \bigsqcup_{i=1}^{n}\left(s_{i}, t_{i}\right] .
$$

Then for every $\varepsilon>0$ there is a map $g: T \rightarrow T$ such that the following hold:

(a) $g$ is a $Q$-linear Markov map, where $Q \cap R=P$;

(b) $g$ is exactly Devaney chaotic;

(c) $(1 / n) \log 2-\varepsilon<h(g)<\max \{h(f),(1 / n) \log 2\}+\varepsilon$;

(d) $g(x)=f(x)$ for every $x \in R \backslash\left(s_{0}, s_{n}\right]$.

Proof. Endow $T$ with a convex metric. For $i=1, \ldots, n$ put $A_{i}=\left[s_{i}, t_{i}\right]$ and define an order on $A_{i}$ in such a way that $s_{i}<t_{i}$. Denote the $P$-basic arcs of $f$ by $B_{1}, \ldots, B_{p}$, where $B_{p}=\left[s_{0}, s_{n}\right]$. Let $\varphi: I \rightarrow R$ be a continuous surjection such that $\varphi(0)=\varphi(1)=s_{1}$ and, for some $m \in \mathbb{N}, \varphi$ maps linearly every $[(l-1) / m, l / m]$ onto some $B_{j_{l}}$.

Fix an integer $N>6$. In every $\left[s_{i}, t_{i}\right]$ choose points $t_{i}^{j}(j=0,1, \ldots, N)$ in such a way that $s_{i}=t_{i}^{0}<t_{i}^{1}<\cdots<t_{i}^{N}=t_{i}$; for $j=1, \ldots, N$ put $A_{i}^{j}=\left[t_{i}^{j-1}, t_{i}^{j}\right]$. Moreover, take points $t_{n}^{N-2}=t_{n}^{N-1,0}<t_{n}^{N-1,1}<\cdots<t_{n}^{N-1, m}=t_{n}^{N-1}$ in $A_{n}^{N-1}$ and put $A_{n}^{N-1, l}=\left[t_{n}^{N-1, l-1}, t_{n}^{N-1, l}\right]$ for $l=1, \ldots, m$.

Put

$$
Q=P \sqcup\left\{t_{i}^{j}: 1 \leq i \leq n, 1 \leq j \leq N\right\} \sqcup\left\{t_{n}^{N-1, l}: 1 \leq l<m\right\}
$$

and define a map $g=g_{N}: T \rightarrow T$ as follows (see Figure 1):

- $g(x)=f(x)$ for $x \in R \backslash\left(s_{0}, s_{n}\right]$;

- $g\left(t_{i}^{j}\right)=t_{i+1}^{j}$ for every $i<n$ and $j \geq 1$;

- $g\left(t_{n}^{j}\right)= \begin{cases}t_{1}^{j+1} & \text { if } j=0,1, \ldots, N-6 ; \\ t_{1}^{N-1} & \text { if } j=N-5, N-3 ; \\ t_{1}^{N} & \text { if } j=N-4 ; \\ t_{1}^{0}=s_{1} & \text { if } j=N-2, N-1 ; \\ t_{1}^{1} & \text { if } j=N ;\end{cases}$

in particular, $g\left(s_{n}\right)=g\left(t_{n}^{0}\right)=t_{1}^{1}$; 


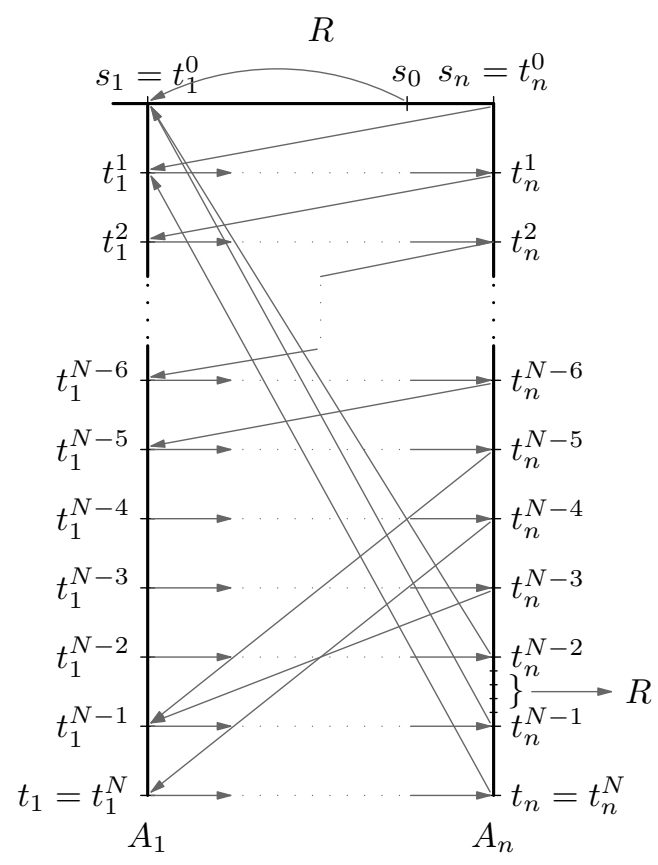

FiguRE 1. The map $g=g_{N}: T \rightarrow T$ (here $R$ is an arc)

- $g\left(t_{n}^{N-1, l}\right)=\varphi(l / m)$ for every $1 \leq l<m$;

- $g$ is $Q$-linear.

Obviously, the map $g$ satisfies (d), it is a $Q$-linear Markov map and $Q \cap R=P$, so (a) is also satisfied. To prove (b) and (c) we need to describe the transition graph of $g$. The $Q$-basic arcs are:

$$
\begin{gathered}
B_{i} \quad(1 \leq i \leq p), \quad A_{i}^{j} \quad(1 \leq i \leq n, 1 \leq j \leq N,(i, j) \neq(n, N-1)) \\
\text { and } \quad A_{n}^{N-1, l} \quad(1 \leq l \leq m) .
\end{gathered}
$$

The transition graph $G_{g}$ of $g$ is as follows (see Figure 2):

- $B_{i} \stackrel{g}{\longrightarrow} B_{j}$ if and only if $B_{i} \stackrel{f}{\longrightarrow} B_{j}$ for every $i<p$ and every $j$;

- $B_{p} \stackrel{g}{\longrightarrow} A_{1}^{1}$;

- $A_{i}^{j} \stackrel{g}{\longrightarrow} A_{i+1}^{j}$ if $i<n$ and $(i, j) \neq(n-1, N-1)$;

- $A_{n-1}^{N-1} \stackrel{g}{\longrightarrow} A_{n}^{N-1, l}$ for every $l=1, \ldots, m$;

- $A_{n}^{j} \stackrel{g}{\longrightarrow} \begin{cases}A_{1}^{j+1} & \text { if } j \leq N-6 ; \\ A_{1}^{N-k}(k=1, \ldots, 4) & \text { if } j=N-5 ; \\ A_{1}^{N} & \text { if } j=N-4, N-3 ; \\ A_{1}^{k}(k=1, \ldots, N-1) & \text { if } j=N-2 ; \\ A_{1}^{1} & \text { if } j=N ;\end{cases}$

- $A_{n}^{N-1, l} \stackrel{g}{\longrightarrow} B_{j_{l}}$ for every $l=1, \ldots, m$.

Now we prove (b). Since there is a path from every $B_{i}$ to $B_{p}(f$ is $(P, S)$ linear), $\varphi$ is a surjection (so $\left\{j_{1}, \ldots, j_{m}\right\}=\{1, \ldots, p\}$ ) and there is a path from $B_{p}$ to any $A_{i}^{j}, A_{n}^{N-1, l}$, we have that the transition graph $G_{g}$ is irreducible. But 


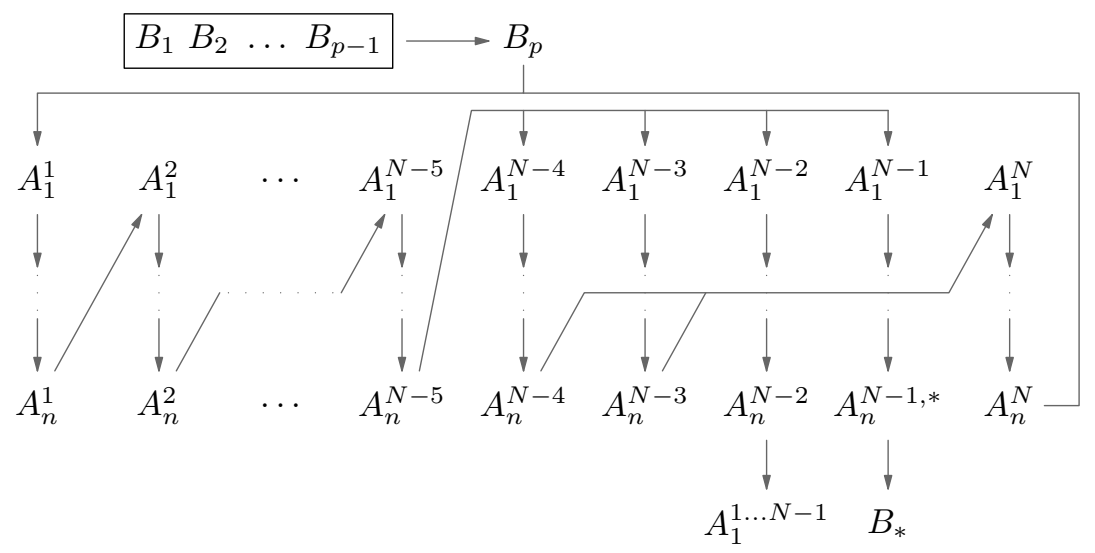

FiguRE 2. The transition graph of $g$

we even have that $G_{g}$ is primitive since there are two loops over $B_{p}$ the lengths of which differ by 1 . Indeed, take a loop through some $A_{n}^{N-1, l}$ such that $j_{l}=p$; then take $l^{\prime}$ such that $B_{j_{l^{\prime}}} \stackrel{g}{\longrightarrow} B_{p}, j_{l^{\prime}}<p$ (notice that $p \geq 2$ since $n \geq 2$ ) and construct another loop by replacing the subpath $A_{n-1}^{N-1} \stackrel{g}{\longrightarrow} A_{n}^{N-1, l} \stackrel{g}{\longrightarrow} B_{p}$ by $A_{n-1}^{N-1} \stackrel{g}{\longrightarrow} A_{n}^{N-1, l^{\prime}} \stackrel{g}{\longrightarrow} B_{j_{l^{\prime}}} \stackrel{g}{\longrightarrow} B_{p}$. So $g$ is an exact map; since on trees transitivity implies dense periodicity we have that $g$ is exactly Devaney chaotic.

The rest of the proof is devoted to (c). Recall that $g=g_{N}$ depends on $N$. To prove the upper bound in (c) it suffices to show that $\lim _{\sup _{N \rightarrow \infty}} h\left(g_{N}\right) \leq$ $\max \{h(f),(1 / n) \log 2\}$. Let $\lambda_{f}$ be the Perron eigenvalue of the transition matrix of $f$. Let $M_{g_{N}}$ be the transition matrix of $g_{N}$ and $\lambda_{g_{N}}$ be the Perron eigenvalue of $M_{g_{N}}$. Since $M_{g_{N}}$ is primitive we have that $\lambda_{g_{N}}>1$. Let $M_{N}$ be the 01-matrix created from $M_{g_{N}}$ by replacing 0's with 1's in the positions corresponding to the edges from $B_{p}$ to $B_{i}$ provided $B_{p} \stackrel{f}{\longrightarrow} B_{i}$. If $\lambda_{N}$ denotes the Perron eigenvalue of $M_{N}$ then, since $M_{g_{N}} \leq M_{N}$,

$$
h\left(g_{N}\right)=\log \lambda_{g_{N}} \leq \log \lambda_{N} .
$$

Since every loop in $G_{g}$ which is not a subset of $\left\{B_{1}, \ldots, B_{p}\right\}$ passes $A_{n}^{1}$ or $A_{n}^{N-2}$, the set

$$
\mathcal{R}=\left\{B_{1}, \ldots, B_{p} ; B_{p+1}=A_{n}^{1}, B_{p+2}=A_{n}^{N-2}\right\}
$$

of $Q$-basic arcs is a rome for $M_{N}$. Notice that trivially $\left\{B_{1}, \ldots, B_{p}\right\}$ is a rome for the transition matrix $M_{f}$ of $f$. For $i, j=1, \ldots, p+2$ put

$$
r_{i j}=r_{i j}(x, N)=\sum_{\pi} \frac{1}{x^{|\pi|}}
$$

where the summation is over all $\mathcal{R}$-simple paths $\pi$ from $B_{i}$ to $B_{j}$. Since for $i, j \leq$ $p$ any simple path from $B_{i}$ to $B_{j}$ visits only $\operatorname{arcs} B_{1}, \ldots, B_{p}$, for $i, j \leq p$ the quantity $r_{i j}$ does not depend on $N$. Let $R_{N}(x)$ and $R_{f}(x)$ denote the matrices $\left(r_{i j}(x, N)\right)_{i, j=1}^{p+2}$ and $\left(r_{i j}(x)\right)_{i, j=1}^{p}$, respectively. Define also

$$
\Phi_{N}(x)=\operatorname{det}\left(R_{N}(x)-E\right) \quad \text { and } \quad \Phi_{f}(x)=\operatorname{det}\left(R_{f}(x)-E\right)
$$


where $E$ denotes the identity matrix of the corresponding dimension. Then $\lambda_{N}$ and $\lambda_{f}$ are the largest positive roots of $\Phi_{N}$ and $\Phi_{f}$, respectively (see Subsection 2.1). Our aim is to show that

$$
\limsup _{N \rightarrow \infty} \lambda_{N} \leq \max \left\{\lambda_{f}, \sqrt[n]{2}\right\}
$$

To this end we must first estimate $r_{i j}=r_{i j}(x, N)$.

Realize that

$$
r_{i, p+1}=0 \quad \text { for } i<p, \quad r_{p, p+1}=x^{-n} \quad \text { and } \quad r_{i, p+2}=0 \quad \text { for } i \leq p,
$$

since every path from $B_{i}(i \leq p)$ to $B_{p+1}=A_{n}^{1}$ or to $B_{p+2}=A_{n}^{N-1}$ must pass both $B_{p}$ and $B_{p+1}$; hence the only such path which is simple is the path $B_{p} \rightarrow A_{1}^{1} \rightarrow$ $A_{2}^{1} \cdots \rightarrow A_{n}^{1}=B_{p+1}$ and the length of it is $n$.

The simple paths starting from $B_{p+1}=A_{n}^{1}$ to any member of $\mathcal{R}$ are "long" (their lengths are at least $n(N-5))$ and their number does not depend on $N$. Indeed, there are $m$ simple paths from $B_{p+1}$ to some of $B_{i}$ 's $(i \leq p)$, two simple loops over $B_{p+1}$ and one simple path from $B_{p+1}$ to $B_{p+2}$. Hence

$$
r_{p+1, j} \in \mathcal{P}_{\text {small }} \quad \text { for every } j=1, \ldots, p+2 .
$$

There are exactly $(N-3)$ simple loops over $B_{p+2}$; indeed, for any $j=2, \ldots, N-2$ there is exactly one which starts with $B_{p+2} \rightarrow A_{1}^{j}$ and the length of it is $n(N-1-j)$. Hence, for $x>1$,

$$
r_{p+2, p+2}=\frac{1}{x^{n}}+\frac{1}{x^{2 n}}+\cdots+\frac{1}{x^{(N-3) n}}=\frac{1}{x^{n}-1}+o,
$$

where $o \in \mathcal{P}_{\text {small }}$. Finally,

$$
r_{p+2, i} \in \mathcal{P} \quad \text { for every } i \leq p+1 .
$$

Now we are ready to estimate $\Phi_{N}(x)=\operatorname{det}\left(R_{N}(x)-E\right)$. Expanding the determinant along the $(p+1)$-st row, then along the last column and using (3.7), (3.6), (3.8), (3.9) and Lemma 18 give (with $o, o^{\prime}, o^{\prime \prime} \in \mathcal{P}_{\text {small }}$ )

$$
\Phi_{N}(x)=(-1) \cdot\left[-1+\frac{1}{x^{n}-1}+o\right] \cdot \operatorname{det}\left(R_{f}(x)-E\right)+o^{\prime}=\frac{x^{n}-2}{x^{n}-1} \cdot \Phi_{f}(x)+o^{\prime \prime} .
$$

Recall that $\lambda_{N}$ denotes the largest positive root of $\Phi_{N}(x)$. Put $\lambda=\lim \sup \lambda_{N}$; without loss of generality we may assume that $\lambda_{N} \rightarrow \lambda$ for $N \rightarrow \infty$. We want to show that $\lambda \leq \tilde{\lambda}:=\max \left\{\lambda_{f}, \sqrt[n]{2}\right\}$. Assume, on the contrary, that $\lambda>\tilde{\lambda}$; so there is $N_{0} \in \mathbb{N}$ and $\delta>0$ such that $\lambda_{N} \geq \tilde{\lambda}+\delta$ for every $N \geq N_{0}$. Put $\psi(x, N)=\Phi_{N}(x)-\frac{x^{n}-2}{x^{n}-1} \cdot \Phi_{f}(x)$ and take $\varepsilon>0$. Since $\psi \in \mathcal{P}_{\text {small }}$, by Lemma 18 there is $N_{1} \in \mathbb{N}$ such that

$$
x^{N / 2} \cdot|\psi(x, N)|<\varepsilon \quad \text { for every } N \geq N_{1} \text { and every } x \geq \tilde{\lambda} .
$$

On the other hand, $\Phi_{f}(x) \neq 0$ for $x>\lambda_{f}$; since $x^{p} \Phi_{f}(x)$ is a polynomial by (2.1), there is $c>0$ with $x^{p}\left|\Phi_{f}(x)\right| \geq c$ for every $x \geq \tilde{\lambda}+\delta$. Thus for every $N \geq N_{0}$ we have

$$
\lambda_{N}^{N / 2} \cdot\left|\psi\left(\lambda_{N}, N\right)\right|=\lambda_{N}^{N / 2} \cdot\left|0-\frac{\lambda_{N}^{n}-2}{\lambda_{N}^{n}-1} \cdot \Phi_{f}\left(\lambda_{N}\right)\right| \geq c \lambda_{N}^{N / 2-p} \cdot \frac{\lambda_{N}^{n}-2}{\lambda_{N}^{n}-1},
$$

which goes to infinity for $N \rightarrow \infty$; this contradicts (3.10). Hence we have proved (3.5). Since $h(f)=\log ^{+} \lambda_{f}$, the upper bound in (c) for $g=g_{N}$ with large $N$ follows from (3.4). 
To prove the lower bound in (c), let $\tilde{M}_{N} \leq M_{g_{N}}$ be the 01-matrix obtained from $M_{g_{N}}$ by keeping only those 1's which correspond to paths $A \rightarrow B$ with $A, B \in\left\{A_{i}^{j}\right.$ : $i \leq n, j \leq N-2\}$; all other entries of $M_{g_{N}}$ are replaced by 0 . Then $\tilde{\mathcal{R}}=\left\{A_{n}^{N-2}\right\}$ is a rome for $\tilde{M}_{N}$ and the corresponding matrix function is

$$
R_{\tilde{\mathcal{R}}}=\left(\tilde{r}_{11}\right), \quad \tilde{r}_{11}(x, N)=\frac{1}{x^{n}}+\frac{1}{x^{2 n}}+\cdots+\frac{1}{x^{(N-4) n}} .
$$

By a simple analysis we can show that the largest positive root $\tilde{\lambda}_{N}$ of $\operatorname{det}\left(R_{\tilde{\mathcal{R}}}(x)-\right.$ $E)=\tilde{r}_{11}(x)-1$ converges to $\sqrt[n]{2}$. Thus $\liminf \lambda_{g_{N}} \geq \lim \tilde{\lambda}_{N}=\sqrt[n]{2}$. Now also the lower bound in (c) for $g=g_{N}$ with large $N$ follows.

\section{EXACT SMALL ENTROPY MAPS ON DENDRITES}

Here we prove the main results of the paper stated in the introduction. They will follow from the next proposition.

Proposition 20. Let $X$ be a non-degenerate dendrite and let $T \subseteq X$ be a tree with end points $t_{1}, \ldots, t_{n}$ and with every terminal edge having non-empty interior in $X$. If there exists a map $f: T \rightarrow T$ which is $(P, S)$-linear for some $P$ and $S=\left(s_{0}, t_{1}, \ldots, t_{n}\right)$ (where $\left.s_{0} \in P\right)$, then

$$
I^{\mathcal{E D}}(X) \leq \max \{h(f),(1 / n) \log 2\} .
$$

Before going into the details of the proof, let us outline the main steps of it. We write $T$ in the form $T=R \sqcup \bigsqcup_{i=1}^{n}\left(s_{i}, t_{i}\right]$, where $R$ is homeomorphic to $T$, $\operatorname{arcs}$ $\left[s_{i}, t_{i}\right]$ have non-empty interiors and $s_{i}, t_{i}$ are end points of $R, T$, respectively. We may assume that the $(P, S)$-linear map $f$ is defined on $R$ and $S=\left(s_{0}, s_{1}, \ldots, s_{n}\right)$ for some $s_{0} \in P$. Using Proposition [19] we construct an exactly Devaney chaotic $Q$-linear map $g: T \rightarrow T$ with $h(g)<\max \{h(f),(1 / n) \log 2\}+\varepsilon$.

Using the $Q$-basic $\operatorname{arcs} A$ of $T$ we construct a splitting $\tilde{\mathcal{A}}$ of $X$ into subcontinua $\tilde{A}$ in such a way that most of the members of $\tilde{\mathcal{A}}$ are free arcs; only subcontinua $\tilde{A}$ corresponding to $Q$-basic $\operatorname{arcs} A$ for which either $A \subseteq R$ or $A \ni t_{i}$, may be "complicated" (that is, they need not be free arcs).

Then we construct a map $F: X \rightarrow X$ in three steps, see (4.10). First, on most of the subcontinua $\tilde{A}$ which are free arcs we put $F(x)=g(x)$. Second, for subcontinua $\tilde{A}$ which either contain an end point of $T$ or are not subsets of $R$ and $g(A)$ is a subset of some "complicated" $\tilde{B}$ (see the definition of $\mathcal{D}$ in (4.2)), we use Propositions 7 and 8 to define $F$ in such a way that the restrictions $\left.F\right|_{\tilde{A}}$ are LEL-maps. Finally, on the remaining "complicated" subcontinua $\tilde{A}$, (that is, for those $\tilde{A}$ with $A$ being a $Q$-basic arc of $R$ ), we define $F$ to be the composition of $g$ with a Lipschitz-1 retraction of $\tilde{A}$ onto $A$, obtained from Lemma 9

During the construction of $F$ we also define a convex metric $d$ on $X$ (see (4.7)), mainly using Lemma14(to obtain a convex metric $d_{T}$ on $T$ such that $g$ has constant slope) and Proposition 7 (to obtain a convex metric on "complicated" subcontinua $\tilde{A})$. Then we prove that the map $F$ is $Q$-Lipschitz w.r.t. this metric and the splitting $\tilde{\mathcal{A}}$, see (4.11). Using properties of $g$, mainly the inequality (4.3), and the fact that the transition matrices $M_{F}$ and $M_{g}$ of $F$ and $g$ coincide, Proposition 11 gives us that the entropy of $F$ can be only slightly larger than that of $g$, see (4.12). Finally, we prove that $F$ is exactly Devaney chaotic. 
Proof. Let $X$ be a dendrite and let $T \subseteq X$ be a tree with end points $t_{1}, \ldots, t_{n}$ and with every terminal edge having non-empty interior in $X$. So we may write

$$
T=R \sqcup \bigsqcup_{i=1}^{n}\left(s_{i}, t_{i}\right]
$$

where $R$ is homeomorphic to $T, E(R)=\left\{s_{1} \ldots, s_{n}\right\}$ and for every $i$ there is $u_{i} \in$ $\left(s_{i}, t_{i}\right)$ such that $\left[s_{i}, u_{i}\right]$ is a free $\operatorname{arc}$ in $X$. By the assumption there are $P \subseteq R$ and a $(P, S)$-linear Markov map $f: R \rightarrow R$, where $S=\left(s_{0}, s_{\pi(1)}, \ldots, s_{\pi(n)}\right)$ for some $s_{0} \in R$ and a permutation $\pi$ of $\{1, \ldots, n\}$. By reordering the indices, if necessary, we may assume that $\pi$ is the identity, that is, $S=\left(s_{0}, s_{1}, \ldots, s_{n}\right)$.

Let $N>6$, let $g=g_{N}: T \rightarrow T$ be the map constructed in the proof of Proposition 19 and $\lambda=\lambda_{N}$ be such that $h(g)=\log \lambda$; then

$$
\limsup _{N \rightarrow \infty} \lambda_{N} \leq \exp (\max \{h(f),(1 / n) \log 2\}) .
$$

We will keep the notation from the proof of Proposition 19 (see e.g. Figures 1 and 2); without loss of generality we may assume that $u_{i}=t_{i}^{N-1}$ for every $i$. Let $\mathcal{A}$ be the set of all $Q$-basic arcs of $g$, see (3.3). Put

$$
\mathcal{D}=\left\{A_{i}^{N}, A_{n}^{N-4}, A_{n}^{N-3}, A_{n}^{N-1, l}: i \leq n, l \leq m\right\}, \quad \mathcal{B}=\mathcal{A} \backslash \mathcal{D} .
$$

We first prove that

$$
\theta_{\mathcal{B}} \leq \frac{2}{N-5}
$$

where $\theta_{\mathcal{B}}=\lim \sup _{h \rightarrow \infty}\left(k_{h} / h\right), k_{h}=\max _{\mathbb{C}} \#\left\{i<h: C_{i} \notin \mathcal{B}\right\}$ and the maximum is taken over all $\mathbb{C}=\left(C_{0}, \ldots, C_{h-1}\right) \in \mathcal{A}^{h}$. To this end take $h \in \mathbb{N}$ and $\left(C_{0}, \ldots, C_{h-1}\right) \in \mathcal{A}^{h}$. If $i<h$ is such that $C_{i} \in \mathcal{D}$ then there are $l \in\{i, \ldots, i+n\}$ and $l^{\prime} \geq \min \{h-1, l+n(N-5)\}$ such that

$$
C_{j} \in \mathcal{B} \quad \text { for every } l<j \leq l^{\prime} .
$$

(Indeed, to define $l$, distinguish two cases: if $g\left(C_{i}\right) \subseteq R$ put $l=i$; otherwise $g^{n^{\prime}}\left(C_{i}\right)=A_{1}^{1}$ for some $1 \leq n^{\prime} \leq n+1$ and put $l=i+n^{\prime}-1$. If there is no $h>i$ with $C_{h} \in \mathcal{D}$, put $l^{\prime}=h-1$. Otherwise let $l^{\prime} \geq i$ be the smallest integer such that $C_{l^{\prime}+1} \in \mathcal{D}$. Then the path $\left(C_{l+1}, C_{l+2}, \ldots, C_{l^{\prime}}\right)$ contains subpath $\left(A_{1}^{1}, \ldots, A_{n}^{1} ; A_{1}^{2}, \ldots, A_{n}^{2} ; \ldots ; A_{1}^{N-5}, \ldots, A_{n}^{N-5}\right)$; hence $l^{\prime}-l \geq n(N-5)$.)

Denote by $i_{1}<\cdots<i_{k}$ those indices $i$ for which $C_{i} \in \mathcal{D}$. Then the previous observation easily implies that $i_{j+n+1}-i_{j}>n(N-5)$ for every $j<k-n$. Hence $h>i_{1+r(n+1)}-i_{1}>r n(N-5)$, where $r=\lfloor(k-1) /(n+1)\rfloor$. Thus $(k-1) /(n+1)-1<$ $r<m /(n(N-5))$ and so

$$
\frac{k_{h}}{h}<\frac{2}{N-5}+\frac{n+2}{h} .
$$

From this (4.3) follows.

To construct our $Q$-Lipschitz map $F: X \rightarrow X$, we first define a splitting $\tilde{\mathcal{A}}=$ $\{\tilde{A}: A \in \mathcal{A}\}$ of $X$. For every $t \in T$ we denote by $X_{t}$ the union of $\{t\}$ and of all components of $X \backslash\{t\}$ disjoint with $T$. For any subset $M$ of $T$ put

$$
X_{M}=\bigsqcup_{t \in M} X_{t}
$$


For every $\operatorname{arc} A \in \mathcal{A}$ we define a subcontinuum $\tilde{A}$ of $X$ in such a way that the following hold:

- the system $\tilde{\mathcal{A}}=\{\tilde{A}: A \in \mathcal{A}\}$ is a splitting of $X$ into continua;

- $\tilde{A} \supseteq A$, and $\tilde{A}=A$ for every $A=A_{i}^{j}$ with $j \neq N$ and for every $A=A_{n}^{N-1, l}$;

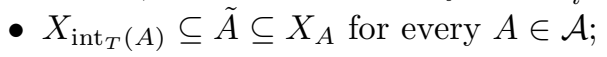

- for every $t \in T$ there is $A \in \mathcal{A}$ such that $X_{t} \subseteq \tilde{A}$;

- if $A \neq B$ then $\tilde{A} \cap \tilde{B}=A \cap B$; hence $P_{\tilde{\mathcal{A}}}=\bigcup_{\tilde{A} \neq \tilde{B}}(\tilde{A} \cap \tilde{B}) \subseteq Q$.

Notice that if $A=[t, u] \in \mathcal{A}$ then $\tilde{A}$ is one of the following four sets: $X_{(t, u)} \sqcup\{t\} \sqcup\{u\}$, $X_{(t, u)} \sqcup X_{t} \sqcup\{u\}, X_{(t, u)} \sqcup\{t\} \sqcup X_{u}, X_{A}=X_{(t, u)} \sqcup X_{t} \sqcup X_{u}$.

Now we define a convex metric $d=d_{N}$ on $X$. Let $d_{T}$ be a convex metric on $T$ such that

$$
g:\left(T, d_{T}\right) \rightarrow\left(T, d_{T}\right) \quad \text { is } Q \text {-linear with constant slope } \lambda=\lambda_{N}>1,
$$

see Lemma 14, Put $l_{A}=\mathcal{H}_{d_{T}}^{1}(A)$ for $A \in \mathcal{A}$; then

$$
\mathcal{H}_{d_{T}}^{1}(g(A))=\sum_{B: A \stackrel{g}{\rightarrow} B} l_{B}=\lambda \cdot \mathcal{H}_{d_{T}}^{1}(A)=\lambda \cdot l_{A} \quad \text { for every } A \in \mathcal{A} .
$$

For $A=\left[a_{0}, a_{1}\right] \in \mathcal{A}$ let $d_{\tilde{A}}=d_{\tilde{A}, a_{0}, a_{1}}$ and $\mathcal{C}_{\tilde{A}}=\mathcal{C}_{\tilde{A}, a_{0}, a_{1}}$ be a convex metric on $\tilde{A}$ and a dense system of subcontinua of $\tilde{A}$ obtained from Proposition 7 (with $A_{1}=A$ ); by (e) we may assume that $\left.d_{T}\right|_{A \times A}=\left.c_{A} \cdot d_{\tilde{A}}\right|_{A \times A}$ for some $c_{A}>0$. If we put $\tilde{l}_{A}=\mathcal{H}_{d_{\tilde{A}}}^{1}(A)$, then $c_{A}=l_{A} / \tilde{l}_{A}$ and $c_{A} \in\left[l_{A}, 2 l_{A}\right]$ by Proposition 7 (a) and the choice of $q$. Let $d$ be the unique convex metric on $X$ with

$$
\left.d\right|_{\tilde{A} \times \tilde{A}}=c_{A} \cdot d_{\tilde{A}} \quad \text { for every } A \in \mathcal{A} .
$$

Notice that $X$ has finite length:

$$
\mathcal{H}_{d}^{1}(X)=\sum_{A \in \mathcal{A}} c_{A}<\infty .
$$

Now we can define the map $F: X \rightarrow X$. First, for $A \in \mathcal{B}, A \nsubseteq R$ we have that $\tilde{A}=A$ and $\tilde{B}=B$ for every $B$ with $A \stackrel{g}{\rightarrow} B$; in this case we put $F_{A}=\left.g\right|_{A}$. Second, if $A \in \mathcal{B}$ and $A \subseteq R$, we put $F_{A}=g \circ r_{A}$, where $r_{A}: \tilde{A} \rightarrow A$ is a $(\gamma, 1)$-LEL* retraction from Lemma 9. Third, if $A=\left[a_{0}, a_{1}\right] \in \mathcal{D}$, take the unique $Q$-basic arc $B=\left[b_{0}, b_{1}\right] \in \mathcal{A}\left(b_{i}=g\left(a_{i}\right)\right.$ for $\left.i=0,1\right)$ with $A \stackrel{g}{\rightarrow} B$. Let $f_{A}:\left(\tilde{A}, d_{\tilde{A}}\right) \rightarrow\left(\tilde{B}, d_{\tilde{B}}\right)$ be a $\left(2, L_{2}\right)$-LEL map obtained from Proposition 8 and define

$$
F_{A}:(\tilde{A}, d) \rightarrow(\tilde{B}, d), \quad F_{A}(x)=f_{A}(x) \quad \text { for } x \in \tilde{A}
$$

(here $d$ denotes the corresponding restriction of the metric). By (4.6), $l_{B}=\lambda l_{A}$; thus $\operatorname{Lip}\left(F_{A}\right)=\left(l_{B} / l_{A}\right) \cdot\left(\tilde{l}_{A} / \tilde{l}_{B}\right) \cdot \operatorname{Lip}\left(f_{A}\right) \leq 2 \lambda L_{2}$ and $F_{A}$ is length-expanding w.r.t. the constant $\left(l_{B} / l_{A}\right) \cdot\left(\tilde{l}_{A} / \tilde{l}_{B}\right) \cdot 2 \geq \lambda$. Thus,

$$
F_{A} \text { is }\left(\lambda, 2 \lambda L_{2}\right) \text {-LEL. }
$$

Finally, define $F=F_{N}: X \rightarrow X$ by

$$
F(x)=F_{A}(x) \quad \text { for } x \in \tilde{A}, A \in \mathcal{A} .
$$

For $A \in \mathcal{A}$ put $L_{\tilde{A}}=\lambda$ if $A \in \mathcal{B}$ and $L_{\tilde{A}}=2 \lambda L_{2}$ if $A \in \mathcal{D}$. Using (4.9) and (4.5) we have that

$$
F \text { is a } Q \text {-Lipschitz map w.r.t. } \tilde{\mathcal{A}},\left(L_{\tilde{A}}\right)_{\tilde{A} \in \tilde{\mathcal{A}}} \quad \text { and } \quad M_{F}=M_{g} \text {. }
$$


Concerning the entropy of $F=F_{N}$ we use Proposition 11. Since $M_{F_{N}}=M_{g_{N}}$ and $\lambda=\lambda_{N}$ is the maximal eigenvalue of $M_{g_{N}}$, Proposition 11 and (4.3) give

$$
h\left(F_{N}\right) \leq \log \lambda_{N}+\frac{4 \log \left(2 \lambda_{N} L_{2}\right)}{N-5} .
$$

Since $L_{2}$ does not depend on $N$, (4.1) gives

$$
\limsup _{N \rightarrow \infty} h\left(F_{N}\right) \leq \limsup _{N \rightarrow \infty} \log \lambda_{N} \leq \max \{h(f),(1 / n) \log 2\} .
$$

Now we show that $F=F_{N}$ is exactly Devaney chaotic provided $N$ is sufficiently large. Put $\mathcal{C}=\bigcup_{A \in \mathcal{A}} \mathcal{C}_{\tilde{A}}$ and fix any $C \in \mathcal{C}$. For $k \in \mathbb{N}_{0}$ put $C_{k}=F^{k}(C)$ and take $A_{k} \in \mathcal{A}$ such that $C_{k} \cap \tilde{A}_{k}$ is non-degenerate. Let $h \geq 0$ be such that

$$
C_{k} \subseteq \tilde{A}_{k} \quad \text { for every } k<h .
$$

Then, by (4.5), (4.9) and the choice of $r_{A}(A \in \mathcal{B}, A \subseteq R)$,

$$
\mathcal{H}^{1}\left(C_{h}\right) \geq \gamma^{p_{h}} \cdot \lambda^{h} \cdot \mathcal{H}^{1}(C), \quad p_{h}=\#\left\{k<h: A_{k} \subseteq R \text { and } C_{k} \nsubseteq A_{k}\right\} .
$$

By the definition of $F_{A}$ for $A \subseteq R$ we have that if $A_{k} \subseteq R$ and $C_{k} \nsubseteq \subseteq A_{k}$ for some $0<k<h$, then $A_{k-1}$ is equal to some $A_{n}^{N-1, l}$. Hence, by (4.4),

$$
\frac{p_{h}}{h} \leq \frac{k_{h}+1}{h} \leq \frac{2}{N-5}+\frac{n+3}{h} .
$$

Fix a constant $\theta \in(1 / \lambda, 1)$ and assume that $N$ is so large that $\gamma^{2 /(N-5)}>\theta$. Using (4.14) we have that $\mathcal{H}^{1}\left(C_{h}\right) \geq(\lambda \theta)^{h} \cdot \gamma^{n+3} \cdot \mathcal{H}^{1}(C)$. Since $\mathcal{H}^{1}(C)>0, \lambda \theta>1$ and $X$ has finite length by (4.8), we have that $h$ 's satisfying (4.13) are bounded from above. Hence there is $h_{0}$ such that $C_{h_{0}} \nsubseteq \tilde{A}_{h_{0}}$ and thus

$$
C_{h_{0}} \text { intersects } Q \text { for some } h_{0} \in \mathbb{N} \text {. }
$$

Take a subcontinuum $C^{\prime}$ of $C_{h_{0}}$ such that $C^{\prime} \in \mathcal{C}, C^{\prime} \subseteq \tilde{A}_{h_{0}}$ and $C^{\prime} \cap Q \neq \emptyset$ (this is possible due to (4.15) and the definition of $\mathcal{C}$ ). By analogous arguments we can find $h^{\prime}>0$ such that $C^{\prime \prime}=F^{h^{\prime}}\left(C^{\prime}\right)$ is not a subset of any $\tilde{A}$. We may assume that $h^{\prime}$ is the smallest integer with this property, hence $F^{h^{\prime}-1}\left(C^{\prime}\right) \subseteq \tilde{A}$ for some $A \in \mathcal{A}$. By inspecting the transition graph of $g$ we see that either $A \subseteq R$ or $A \in\left\{A_{n}^{N-5}, A_{n}^{N-2}, A_{n-1}^{N-1, l}: l=1, \ldots, m\right\}$. Since $F^{h^{\prime}-1}\left(C^{\prime}\right)$ intersects $Q$, the set $C^{\prime \prime}$ contains a $Q$-basic arc $B$ such that either $B \subseteq R$ or $B$ is a free $\operatorname{arc}$ in $X$. In the former case we have that $F^{k}(B) \supseteq A_{1}^{1}$ for some $k$; so in both cases

there is $h \in \mathbb{N}$ such that the set $C_{h}$ contains some $A \in \mathcal{A}$ with $\tilde{A}=A$.

But now $C_{h+k} \supseteq \bigcup_{g^{k}(A) \supseteq B} \tilde{B}$ for every $k>0$. Since $g$ is exact we have that $C_{h+k}=X$ for every sufficiently large $k$. Hence $F$ is exact. Finally, since $X$ has a free arc which disconnects $X$, by AKLS99, Theorem 1.1] the map $F$, being transitive, has dense periodic points.

To summarize, we have proved that $F_{N}$ is exactly Devaney chaotic for every sufficiently large $N$ and that $\lim \sup _{N \rightarrow \infty} h\left(F_{N}\right) \leq \max \{h(f),(1 / n) \log 2\}$. Thus the proposition is proved.

Now we are ready to prove the results stated in the introduction. 
Proof of Theorem $A$, Let $X$ be a non-degenerate dendrite such that no subtree of it contains all free $\operatorname{arcs}$ of $X$. Fix $n=2^{r}, r \geq 1$ and $\varepsilon>0$. By Lemma 5 , $X$ contains a subtree $T$ which is either an $n$-star or an $n$-comb such that every terminal edge of $T$ has non-empty interior in $X$. Since $T \in \mathcal{T}_{0}$, Lemma 16 gives that there is a $(P, S)$-linear Markov map $f: R \rightarrow R$ with $h(f)<(1 / n) \log 2+\varepsilon$, where $P \subseteq T$ and $S=\left(s_{0}, s_{1}, \ldots, s_{n}\right)$ for some $s_{0} \in T$ and the end points $s_{1}, \ldots, s_{n}$ of $T$. Now Proposition 20 gives that $I^{\mathcal{E D}}(X)<(1 / n) \log 2+\varepsilon$. Since $n=2^{r}$ and $\varepsilon$ are arbitrary, we have $I^{\mathcal{E D}}(X)=0$.

The fact that the infima $I^{\mathcal{T}}(X), I^{\mathcal{E D}}(X)$ are not attainable, follows from DSS12. Indeed, the space $X$, being a compact metric space which is not a finite union of disjoint simple closed curve and which contains a free arc, admits no zero entropy transitive map.

In the proof of Theorem A we have used Lemma [16, which is a reformulation of Lemmas 4.6 and 4.7 from Ye00. The use of Lemma 16 can be replaced by Lemmas 21, 22 which we prove in Appendix 1. This makes the proof of Theorem $\mathrm{A}$ independent of Ye's results.

Proof of Corollary $B$, Let $X$ be a non-degenerate completely regular continuum which is not a tree. If $X$ is a dendrite then $I^{\mathcal{E D}}(X)=0$ by Theorem $\mathrm{A}$, since free arcs are dense in $X$ by Lemma 5. Otherwise $X$ contains a simple closed curve $S$. Since $X$ is completely regular, $S$ has non-empty interior and thus $X$ contains a free arc; of course, this arc does not disconnect $X$. So $I^{\mathcal{E D}}(X)=0$ by Sp12b.

Proof of Proposition C. Just use Proposition 20 and Lemma 16.

\section{Appendix 1: Zero entropy $(P, S)$-Linear Markov maps on Stars And COMBS}

The purpose of this appendix is to give a simple construction of $(P, S)$-linear Markov maps with zero entropy on stars and combs. This makes the proof of Theorem A independent on the construction of Ye00.

Lemma 21. Let $T$ be an $n$-star with end points $s_{1}, \ldots, s_{n}(n \geq 2)$. Then there is a map $f: T \rightarrow T$, a finite subset $P$ of $T$ and $s_{0} \in P$ such that $f$ is a $(P, S)$-linear Markov map with zero-entropy, where $S=\left(s_{0}, s_{1}, \ldots, s_{n}\right)$.

Proof. Let $b$ be the only branch point of $T$; so $T=\bigcup_{i=1}^{n}\left[b, s_{i}\right]$. For every $i$ take a point $s_{i}^{\prime} \in\left(b, s_{i}\right)$. Put $P=\left\{b ; s_{1}^{\prime}, \ldots, s_{n}^{\prime} ; s_{1}, \ldots, s_{n}\right\}, s_{0}=s_{n}^{\prime}$ and $S=\left(s_{0}, s_{1}, \ldots, s_{n}\right)$. Define the $P$-linear Markov map $f$ on $T$ by $f(b)=s_{1}^{\prime}$, $f\left(s_{n}^{\prime}\right)=s_{1}, f\left(s_{n}\right)=s_{1}^{\prime}$ and, for every $1 \leq i<n, f\left(s_{i}^{\prime}\right)=s_{i+1}^{\prime}$ and $f\left(s_{i}\right)=s_{i+1}$ (see Figure 3 for $n=4)$.

Obviously $f$ is $(P, S)$-linear. It is an easy exercise to show that $h(f)=0$. Indeed, one can use e.g. the rome method with the rome consisting of two arcs: $A=\left[b, s_{n}^{\prime}\right]$, $B=\left[s_{n}^{\prime}, s_{n}\right]$. Since there is no path from $B$ to $A$ and the length of every simple path from $A$ to $A$ and from $B$ to $B$ is $n$, the characteristic polynomial of the transition matrix of $f$ is (see (2.1))

$$
(-1)^{2 n-2} \cdot x^{2 n} \cdot\left(\frac{1}{x^{n}}-1\right)^{2}=\left(x^{n}-1\right)^{2},
$$

the largest positive root of which is $\lambda=1$. Hence the entropy of $f$ is $h(f)=\log 1=$ 0 . 


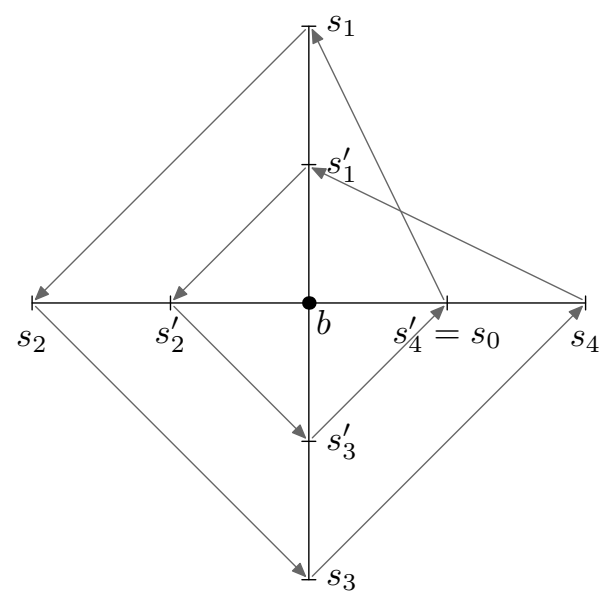

Figure 3. The map $f$ on a 4 -star

Lemma 22. Let $n=2^{r}(r \geq 1)$ and let $T$ be an $n$-comb. Then there are a finite set $P$ and a tuple $S=\left(s_{0}, s_{1}, \ldots, s_{n}\right)$, where $s_{0} \in P$ and $\left\{s_{1}, \ldots, s_{n}\right\}=E(T)$, such that $T$ admits a $(P, S)$-linear map $f: T \rightarrow T$ with zero entropy.

Proof. First we introduce some notation for distinguished points of $T$, which will form $P$. Put $\Sigma=\{0,1\}$ and $\Sigma_{k}^{l}=\bigcup_{i=k}^{l} \Sigma^{i}$ for $0 \leq k \leq l \leq r$, where $\Sigma^{0}$ is the singleton $\{\theta\}$ ( $\theta$ denotes the empty word); that is, $\Sigma_{k}^{l}$ is the set of all words $\alpha=\alpha_{0} \ldots \alpha_{i-1}$ over the alphabet $\Sigma$ of the length $|\alpha|=i \in\{k, \ldots, l\}$. The concatenation $\alpha \beta$ and the powers $\alpha^{k}$ for words $\alpha, \beta$ and integers $k \geq 1$ are defined in a natural way. For every $k$ let $<$ denote the lexicographical ordering on $\Sigma^{k}$. Notice that $\Sigma^{k}$ with the addition from the left to the right is a group; for $\alpha \in \Sigma^{k}$ and $n \in \mathbb{Z}$ we define $\alpha+n$ as usual.

Fix $r \geq 1$ and $n=2^{r}$. Put $a_{\theta}=1 / 2$ and

$$
a_{\alpha}=\frac{\alpha_{0}}{2}+\frac{\alpha_{1}}{2^{2}}+\cdots+\frac{\alpha_{k-1}}{2^{k}}+\frac{1}{2^{k+1}} \quad \text { for } \alpha=\alpha_{0} \ldots \alpha_{k-1} \in \Sigma_{1}^{r-1} .
$$

For $\alpha \in \Sigma_{0}^{r-1}$ put $b_{\alpha 0}=a_{\alpha}-2^{-(r+2)}$ and $b_{\alpha 1}=a_{\alpha}+2^{-(r+2)}$. Notice that the following are true:

(i) $b_{\alpha 0}<a_{\alpha}<b_{\alpha 1}$ for $\alpha \in \Sigma_{0}^{r-1}$;

(ii) $b_{\beta}<b_{\gamma}$ for every $\beta<\gamma$ from $\Sigma_{1}^{r}$ of the same length;

(iii) $b_{\alpha 0 \beta}<b_{\alpha 0}<b_{\alpha 1}<b_{\alpha 1 \beta}$ for every $\alpha \in \Sigma_{0}^{r-2}$ and $\beta \in \Sigma_{1}^{r-|\alpha|-1}$.

Identify the points $a_{\alpha}, b_{\beta} \in(0,1)$ with the points $\left(a_{\alpha}, 0\right),\left(b_{\beta}, 0\right)$ of the Euclidean plane and define $c_{\gamma}=\left(b_{\gamma}, 1\right)$ for $\gamma \in \Sigma^{r}$. Without loss of generality we may assume that the $n$-comb $T$ is given by

$$
T=A \cup \bigcup_{\gamma \in \Sigma^{r}} B_{\gamma},
$$

where $A=\left[b_{0^{r}}, b_{1^{r}}\right]$ and $B_{\gamma}=\left[b_{\gamma}, c_{\gamma}\right]\left(\gamma \in \Sigma^{r}\right)$ are segments; see Figure 4 for an illustration. Put

$$
P=\left\{a_{\alpha}: \alpha \in \Sigma_{0}^{r-1}\right\} \cup\left\{b_{\beta}: \beta \in \Sigma_{1}^{r}\right\} \cup\left\{c_{\gamma}: \gamma \in \Sigma^{r}\right\} .
$$


Obviously, $P \supseteq E(T) \cup B(T)$ since $E(T)=\left\{c_{\gamma}: \gamma \in \Sigma^{r}\right\}$ and $B(T)=\left\{b_{\gamma}: \gamma \in\right.$ $\left.\Sigma^{r}, \gamma \neq 0^{r}, 1^{r}\right\}$. The $P$-basic arcs are

- $A_{\alpha 0}=\left[b_{\alpha 0}, a_{\alpha}\right], A_{\alpha 1}=\left[a_{\alpha}, b_{\alpha 1}\right]$ for $\alpha \in \Sigma_{0}^{r-1}$;

- $B_{\alpha 0}=\left[b_{\alpha 01^{k}}, b_{\alpha 0}\right], B_{\alpha 1}=\left[b_{\alpha 1}, b_{\alpha 10^{k}}\right]$ for $\alpha \in \Sigma_{0}^{r-2}$ and $k=r-|\alpha|-1$;

- $C_{\gamma}=\left[b_{\gamma}, c_{\gamma}\right]$ for $\gamma \in \Sigma^{r}$.

For a $P$-basic arc $D$, we define the level of it, denoted by $\operatorname{lev}(D)$, as the length of its index; e.g. $\operatorname{lev}\left(B_{\alpha 0}\right)=|\alpha 0|$ and $\operatorname{lev}\left(C_{\gamma}\right)=r$. For $1 \leq k \leq r$ denote by $\mathcal{S}_{k}$ the system of all $P$-basic $\operatorname{arcs} D$ with $\operatorname{lev}(D) \geq k$; put $\mathcal{S}_{r+1}=\emptyset$.

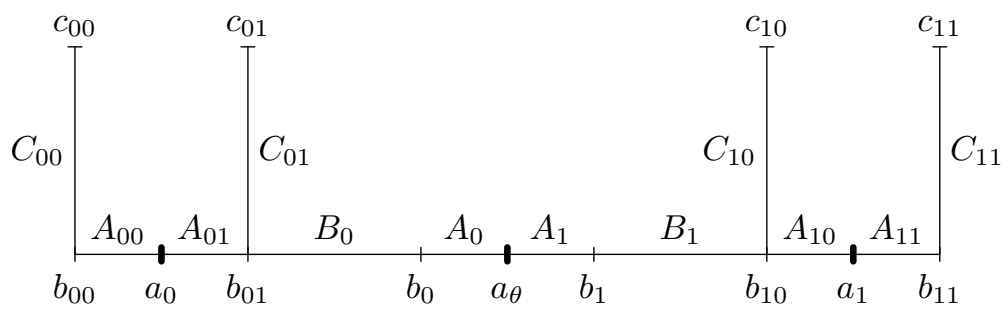

Figure 4 . The $n$-comb $T$ for $n=2^{2}$

Define the map $f: T \rightarrow T$ by

(a) $f\left(a_{\alpha}\right)=a_{\alpha+1}$ for $\alpha \in \Sigma_{0}^{r-1}$;

(b) $f\left(b_{1^{r}}\right)=c_{0^{r}}, f\left(b_{1^{k}}\right)=b_{0^{k+1}}$ for $k<r$ and $f\left(b_{\beta}\right)=b_{\beta+1}$ for $\beta \in \Sigma_{1}^{r} \backslash\left\{1^{k}\right.$ : $1 \leq k \leq r\}$

(c) $f\left(c_{1^{r}}\right)=b_{0^{r}}$ and $f\left(c_{\gamma}\right)=c_{\gamma+1}$ for $\gamma \in \Sigma^{r}, \gamma \neq 1^{r}$;

(d) $f$ is $P$-linear.

A simple analysis shows that the edges of the transition graph $G$ of $f$ are as follows (here we write $D \rightarrow \mathcal{S}_{k}$ if $D f$-covers zero ore more arcs from $\mathcal{S}_{k}, 1 \leq k \leq r+1$ ):

- $A_{\alpha} \rightarrow A_{\alpha+1}$ for $\alpha \notin\left\{1^{k}: k \leq r\right\}$

- $A_{1^{k}} \rightarrow A_{0^{k}}, B_{0^{k}}, A_{0^{k+1}}, \mathcal{S}_{k+1}$ for $k<r$

- $A_{1^{r}} \rightarrow A_{0^{r}}, C_{0^{r}}$

- $B_{\beta} \rightarrow B_{\beta+1}, \mathcal{S}_{|\beta|+1}$ for $\beta \notin\left\{1^{k}: k<r\right\}$;

- $B_{1^{k}} \rightarrow A_{0^{k+1}}, \mathcal{S}_{k+1}$ for $k<r$;

- $C_{\gamma} \rightarrow C_{\gamma+1}$ for every $\gamma$.

Hence the loops of $G$ are

- $A_{0^{k}} \rightarrow A_{0^{k}+1} \rightarrow \cdots \rightarrow A_{1^{k}} \rightarrow A_{0^{k}}, k=1, \ldots, r$

- $C_{0^{r}} \rightarrow C_{0^{r}+1} \rightarrow \cdots \rightarrow C_{1^{r}} \rightarrow C_{0^{r}}$.

Since every $P$-basic arc is contained in at most one loop of $G$, the entropy of $f$ is zero.

Put $S=\left(s_{0}, s_{1}, \ldots, s_{n}\right)$, where $s_{0}=b_{1^{r}}$ and $s_{i}=c_{0^{r}+(i-1)}(i=1, \ldots, n)$. To finish the proof we need to show that $f$ is a $(P, S)$-linear Markov map. Indeed, for $0 \leq i<n$ we have $f\left(s_{i}\right)=s_{i+1}$. Moreover, $s_{n}=c_{1^{r}}$ is an end point of $T$ and $\left[s_{0}, s_{n}\right]=C_{1^{r}}$ is a $P$-basic arc. Finally, from the description of the transition graph of $f$ we see that there is a path from any $P$-basic arc $D$ to $C_{1^{r}}=\left[s_{0}, s_{n}\right]$.

\section{Appendix 2: Trees With LARge numBer of END POINTS}

The lower and upper bounds for the infimum of entropies of transitive systems on a given tree $T$, see ABLM97, Ye00, Ba01, suggest that if the number of end 
points of $T$ is "large" then $T$ admits a transitive map with "small" entropy. In this appendix we show that this is in fact true, even with transitivity replaced by exactness. Moreover, we give an upper bound for the infimum depending only on the number of end points of the tree. This bound is in no sense optimal but it is very simple. (In what follows, log means the natural logarithm.)

Proposition 23. Let $T$ be a tree with $n$ end points. Then

$$
\frac{\log 2}{n} \leq I^{\mathcal{T}}(T) \leq I^{\mathcal{E D}}(T) \leq \frac{\log 2}{\sqrt{\log n}} .
$$

Hence, if $\left(T_{n}\right)_{n}$ is a sequence of trees with the number of end points going to infinity, then

$$
\lim _{n \rightarrow \infty} I^{\mathcal{E D}}\left(T_{n}\right)=0 .
$$

Before proving Proposition 23 we prove the following simple lemma.

Lemma 24. Let $T$ be a tree with $n \geq 3$ end points and such that every branch point of it has order at most $k$. Then $T$ has at least $n / k$ branch points.

Proof. We prove the lemma by induction. If $n=3$ then $T$ is a 3 -star and the assertion is trivial $(k \geq 3$, hence $n / k \leq 1)$. Assume that for some $n>3$ the assertion of the lemma holds for every tree (which is not an arc) with the number of end points strictly smaller than $n$. Let $T$ be a tree with $n$ end points. Take a branch point $b$ of $T$ (there is some since $T$ is not an arc). Let $U_{1}, \ldots, U_{m}(m \leq k)$ be the components of $X \backslash\{b\}$; without loss of generality we may assume that there is an integer $0 \leq m^{\prime} \leq m$ such that $U_{i}$ is not an arc for every $i \leq m^{\prime}$ and $U_{i}$ is an arc for every $i>m^{\prime}$. For every $i \leq m^{\prime}$ put $n_{i}=\# E\left(\overline{U_{i}}\right)$; then $n_{i}<n$ and so the tree $\overline{U_{i}}$ has at least $n_{i} / k$ branch points by the induction hypothesis. Easily we have that $n=\sum_{i=1}^{m^{\prime}}\left(n_{i}-1\right)+\left(m-m^{\prime}\right)$, so $\sum_{i=1}^{m^{\prime}} n_{i} \geq n-\left(m-2 m^{\prime}\right) \geq n-k$. The number of branch points of $T$ is at least $1+\sum_{i=1}^{m^{\prime}} n_{i} / k \geq n / k$.

Proof of Proposition 23. The lower bound from (4.16) was shown in ABLM97. To show the upper bound take any tree $T$ with $n$ end points. If $n=2$ we have $I^{\mathcal{E D}}(T)=(1 / 2) \log 2$, so we may assume that $n \geq 3$. Denote by $k \geq 1$ the only integer such that $\sqrt{\log n}-1 \leq k<\sqrt{\log n}$. By standard techniques one can show that $2 x \cdot \log x<x^{2}$ for $x \geq 1$ (indeed, $f(x)=x^{2}-2 x \cdot \log x$ is strictly increasing on $[1, \infty)$ and $f(1)=1>0)$, hence $k^{2 k}<n$.

If $T$ has a branch point of order at least $k+1$ then it contains a $(k+1)$-star, hence $I^{\mathcal{E D}}(T) \leq \log 2 /(k+1) \leq \log 2 / \sqrt{\log n}$ by Proposition $[\mathrm{C}$ and the choice of $k$. Otherwise every branch point of $T$ has order at most $k$. By Lemma 24 the tree $T$ has at least $n / k>k^{2 k-1}$ branch points, so by Lemma 1 it contains a $(2 k+2)$-comb. Let $r \geq 1$ be the largest integer such that $2^{r} \leq 2 k+2$; hence $2^{r+1}>2 k+2$, that is, $2^{r}>k+1 \geq \sqrt{\log n}$. By Proposition [C] we have that $I^{\mathcal{E D}}(T) \leq \log 2 / 2^{r}<\log 2 / \sqrt{\log n}$. Hence the proof of (4.16) is finished.

\section{REFERENCES}

[ABLM97] L. Alsedà, S. Baldwin, J. Llibre, M. Misiurewicz, Entropy of transitive tree maps, Topology 36 (1997), no. 2, 519-532. MR1415604

[AEO07] G. Acosta, P. Eslami, L. G. Oversteegen, On open maps between dendrites, Houston J. Math. 33 (2007), no. 3, 753-770. MR2335734

[AKLS99] L. Alsedà, S. Kolyada, J. Llibre, L. Snoha, Entropy and periodic points for transitive maps, Trans. Amer. Math. Soc. 351 (1999), no. 4, 1551-1573. MR1451592 
[AKM65] R. L. Adler, A. G. Konheim, M. H. McAndrew, Topological entropy, Trans. Amer. Math. Soc. 114 (1965), 309-319. MR0175106

[ARR99] L. Alsedà, M. A. del Río, J. A. Rodríguez, A splitting theorem for transitive maps, J. Math. Anal. Appl. 232 (1999), no. 2, 359-375. MR1683124

[Ba01] S. Baldwin, Entropy estimates for transitive maps on trees, Topology 40 (2001), no. 3, 551-569. MR1838995

[Ba07] S. Baldwin, Continuous itinerary functions and dendrite maps, Topology Appl. 154 (2007), no. 16, 2889-2938. MR2355878

[BC01] M. Baillif, A. de Carvalho, Piecewise linear model for tree maps, Internat. J. Bifur. Chaos Appl. Sci. Engrg. 11 (2001), no. 12, 3163-3169. MR1885262

[BGMY80] L. Block, J. Guckenheimer, M. Misiurewicz, L. S. Young, Periodic points and topological entropy of one dimentional maps, In Global Theory of Dynamical Systems, Lecture Notes in Mathematics, no. 819, pages 18-34, Springer-Verlag, 1980. MR0591173

[Bi49] R. H. Bing, Partitioning a set, Bull. Amer. Math. Soc. 55 (1949), 1101-1110. MR0035429

[B182] A. Blokh, On sensitive mappings of the interval, Russian Math. Surveys 37 (1982), 203-204. MR650765

[Bl87] A. Blokh, On the connection between entropy and transitivity for one-dimensional mappings, Russ. Math. Surv. 42 (1987), no. 5, 165-166. MR928783

[DSS12] M. Dirbák, L. Snoha, V. Špitalský, Minimality, transitivity, mixing and topological entropy on spaces with a free interval, Ergodic Theory Dynam. Systems (2012), doi:10.1017/S0143385712000442.

[EH43] S. Eilenberg, O. G. Harrold, Continua of finite linear measure I, Amer. J. Math. 65 (1943), 137-146. MR0007643

[HKO11] G. Harańczyk, D. Kwietniak, P. Oprocha, Topological structure and entropy of mixing graph maps, arXiv:1111.0566 1 [math.DS] (2011).

[I198] A. Illanes, A characterization of dendrites with the periodic-recurrent property, Proceedings of the 13th Summer Conference on General Topology and its Applications (México City, 1998). Topology Proc. 23 (1998), Summer, 221-235 (2000). MR1803250

[Ka98] H. Kato, The depth of centres of maps on dendrites, J. Austral. Math. Soc. Ser. A 64 (1998), no. 1, 44-53. MR1490145

[Ma09] E. N. Makhrova, Homoclinic points and topological entropy of a continuous mapping of a dendrite, (Russian) Sovrem. Mat. Prilozh. No. 54, Trudy Mezhdunarodnoi Konferentsii po Dinamicheskim Sistemam i Differentsialnym Uravneniyam. Ch. 2 (2008), 79-86; translation in J. Math. Sci. (N. Y.) 158 (2009), no. 2, 241-248. MR2677136

[Na92] S. B. Nadler, Continuum theory. An introduction, Monographs and Textbooks in Pure and Applied Mathematics, 158, Marcel Dekker, Inc., New York, 1992. MR1192552

[Na12] I. Naghmouchi, Dynamical properties of monotone dendrite maps, Topology Appl. 159 (2012), no. 1, 144-149. MR2852955

[Se81] E. Seneta, Non-negative matrices and Markov chains, Revised reprint of the second (1981) edition [Springer-Verlag, New York; MR0719544]. Springer Series in Statistics. Springer, New York, 2006. MR2209438

[Sp12a] V. Špitalský, Length-expanding Lipschitz maps on totally regular continua, arXiv:1203.2352 [math.DS] (2012).

[Sp12b] V. Špitalský: Entropy and exact Devaney chaos on totally regular continua, to appear in Discrete Contin. Dyn. Syst., arXiv:1112.6017 [math.DS] (2012).

[SWZ10] E.Shi, S. Wang, L. Zhou, Minimal group actions on dendrites, Proc. Amer. Math. Soc. 138 (2010), no. 1, 217-223. MR2550186

[Ye00] X. Ye, Topological entropy of transitive maps of a tree, Ergodic Theory Dynam. Systems 20 (2000), no. 1, 289-314. MR1747021

Department of Mathematics, Faculty of Natural Sciences, Matej Bel University, TAjovského 40, 97401 Banská Bystrica, Slovakia

E-mail address: vladimir.spitalsky@umb.sk 